\title{
Spectroscopic observations of the first helium nova V445 Puppis
}

\author{
T. Iijima ${ }^{1}$ and H. Nakanishi ${ }^{2}$
}

\author{
1 Astronomical Observatory of Padova, Asiago Section, Osservatorio Astrofisico, 36012 Asiago (Vi), Italy \\ e-mail: takashi.iijima@oapd.inaf.it \\ 2 Nobeyama Radio Observatory, 384-1305 Minamimaki, Minamisaku (Nagano), Japan \\ e-mail: hnakanis@nro.nao.ac.jp
}

Received 19 March 2007 / Accepted 15 January 2008

\begin{abstract}
At Asiago Astrophysical Observatory, we observed the peculiar, explosive object Nova (V445) Puppis 2000 during its early decline stage between January and April 2001. We acquired both high and medium dispersion spectra for the optical wavelengths 3900-7000 A. The most remarkable properties of the spectra were an absence of hydrogen lines and unusually strong carbon-ion, emission lines. We identified the most prominent emission line in the optical region at $6585 \AA$ to be due to C II 2,6578 and $6583 \AA$. The second strongest emission line at $5900 \AA$ may be due to Na I D1 and D2, blended by C II 5, 5900 and $5902 \AA$. Prominent emission lines of Fe II, Ti II, Cr II, Si II, Mg II, [Fe II], [Ti II], C I, C II, C III, Ca I, He I, and probably of O II, N II, N III, and S II were in addition detected. Some emission lines of Fe II, Ti II, Cr II, Na I, and Mg II were accompanied by P Cygni type absorption components blue-shifted by about $-500 \mathrm{~km} \mathrm{~s}^{-1}$. Our first spectrum obtained on January 14, 2001 showed an absorption band of CN (cyanogen) molecule at $4215 \AA$. The overall spectral features resembled those of classical slow novae, apart from the absence of hydrogen lines. The appearance of some [Ti II] lines, and a rather high intensity of the emission line of Mg II $23,6545.8 \AA$, were additional peculiar spectral features of this object.

This object may be located in or beyond the Orion arm, because two sets of interstellar absorption components of Na I D1 and D2 were detected. Their radial velocities in the local standard of rest are $+16.0 \pm 0.4 \mathrm{~km} \mathrm{~s}^{-1}$ and $+73.5 \pm 1.0 \mathrm{~km} \mathrm{~s}^{-1}$. The radial velocity of this object is estimated to be $+224 \pm 8 \mathrm{~km} \mathrm{~s}^{-1}$, which suggests that this object belongs to the old disk population. The distance and the interstellar extinction to the object are estimated to be $3.5 \leq d \leq 6.5 \mathrm{kpc}$ and $E(B-V)=0.51 \mathrm{mag}$, respectively. The derived absolute magnitude at light maximum is $-5.8 \leq M_{V} \leq-7.1$ mag. Our results support the classification of this object as a helium nova.
\end{abstract}

Key words. stars: individual: V445 Puppis - novae, cataclysmic variables - ISM: general

\section{Introduction}

Nova (V445) Puppis 2000 was discovered by Kanatsu on December 30,2000 as a star of $m_{\text {vis. }} \cong 8.6$, which was reported by T. Kato (2000). The light curve presented by Ashok $\&$ Banerjee (2003) showed that this object may have reached maximum luminosity $m_{\mathrm{V}} \cong 8.5$ in December 2000 . No sharp peak was seen in the light curve, but it is unclear if the peak was missed, or if there was no peak at all. After a very slow fading of one magnitude in the first six months, a rapid fading began in June 2001 (e.g., Ashok \& Banerjee 2003). From infrared observations it has been suggested that a condensation of dust occurred in the decline stage (Lynch et al. 2001; Ashok \& Banerjee 2003).

The spectra in the early decline stage showed prominent emission lines of C II, C III, Fe II, Na I, Ti II, Cr II, Si II, Mg II, [Fe II], [Ti II], C I, Ca I, He I, but no emission line of H I (Fujii 2001; Wagner et al. 2001a; Kamath \& Anupama 2002; Iijima 2005). Optical (Woudt \& Steeghs 2005) and infrared (Lynch et al. 2004) spectra obtained about three years after the explosion showed the emission lines of He I, [O I], [O II], and [O III], but again no $\mathrm{H}$ I line. The emission lines of highly ionized ions, such as $\mathrm{He}$ II, [Ca V], or [Fe VII], were not detected (Woudt \& Steeghs 2005).

It has been suggested that this object could be a helium nova (Ashok \& Banerjee 2003; Kato \& Hachisu 2003; Kato 2005), that is, its explosion was due to helium burning on a white dwarf. This paper presents high and medium dispersion spectra of V445 Puppis in the early decline stage. Using the observed results, the distance to the object and the interstellar extinction are estimated.

\section{Observation}

Medium dispersion spectra were obtained with a Boller \& Chivens grating spectrograph mounted on the $122 \mathrm{~cm}$ telescope at the Astrophysical Observatory of Asiago, affiliated to the University of Padova. The resolution was $\lambda / \Delta \lambda \cong 800$ with a grating of 600 lines $\mathrm{mm}^{-1}$. High dispersion spectra covering the range $\lambda \lambda 4340-6900 \AA$ with a resolution of $\lambda / \Delta \lambda \cong 8000$ were obtained with an Echelle spectrograph mounted on the $182 \mathrm{~cm}$ telescope at the Mount Ekar station of the Astronomical Observatory of Padova. A log of the observations is given in Table 1.

The spectra were reduced using the standard tasks of the NOAO IRAF ${ }^{1}$ package at the Asiago Observatory. The spectrophotometric calibrations were completed using the spectra of a standard star HD60778 obtained in the same nights. Unfortunately, the errors in the calibrations were large, because all spectra of V445 Pup were obtained with airmass larger than 3.2. Therefore, we were not able to make quantitative arguments

\footnotetext{
1 IRAF is distributed by NOAO for Research in Astronomy, Inc. under cooperative agreement with the National Science Foundation.
} 
Table 1. Log of spectroscopic observations of V445 Pup.

\begin{tabular}{|c|c|c|c|c|c|c|}
\hline \multicolumn{2}{|c|}{ Date } & $\overline{\overline{\mathrm{UT}}}$ & $\overline{\mathrm{JD}}$ & $\begin{array}{r}\text { exp. } \\
\text { s }\end{array}$ & Inst. & $\begin{array}{c}\text { Range } \\
\AA\end{array}$ \\
\hline \multicolumn{7}{|c|}{2001} \\
\hline Jan. & 14 & $23: 15$ & 1924.5 & 1200 & $B \& C$ & $3950-5100$ \\
\hline Jan. & 14 & $23: 58$ & 1924.5 & 600 & " & $5850-7000$ \\
\hline Feb. & 2 & $21: 38$ & 1943.4 & 1800 & $"$ & $4000-5150$ \\
\hline Feb. & 2 & $22: 14$ & 1943.4 & 1200 & " & $4860-6050$ \\
\hline Feb. & 2 & 23:02 & 1943.5 & 1200 & " & $5850-7000$ \\
\hline Feb. & 3 & $22: 11$ & 1944.4 & 2400 & Ech & $4340-6900$ \\
\hline Feb. & 5 & $21: 33$ & 1946.4 & 2400 & "I & $4340-6900$ \\
\hline Feb. & 11 & $21: 16$ & 1952.4 & 1200 & $B \& C$ & $5850-7000$ \\
\hline Feb. & 11 & $21: 56$ & 1952.4 & 1800 & " & $4080-5250$ \\
\hline April & 2 & 19:01 & 2002.3 & 1800 & " & $4060-5230$ \\
\hline
\end{tabular}

JD: Julian date -2450000 . UT: Universal time at start of exposure.

about the intensities of the emission lines in this work. The identifications of the emission and absorption lines were made using the multiplet table of Moore (1959), the catalog of Meinel et al. (1968), and the list in the NIST atomic spectra database ${ }^{2}$.

Wavelengths, equivalent widths, and identifications of selected prominent emission lines and absorption lines, detected in the medium dispersion spectra are given in Table 2. Similar measurements completed for the high dispersion spectrum, acquired on February 3, 2001, are provided in Table 3. The first two values of wavelength in the columns of identifications are omitted. The errors in the equivalent widths may be $\pm 20 \%$ or higher, because many emission lines were blended with others. The $\mathrm{S} / \mathrm{N}$ ratios of the faintest detectable emission features was approximately 10 .

\section{Spectral evolution: medium dispersion spectra}

We present an analysis of the spectral evolution of V445 Pup completed using the medium dispersion spectra. Figure 1 shows the tracings of the blue spectra obtained in the period from January 14 to April 2, 2001. The ordinate is an arbitrary intensity scale. The identifications of the prominent emission and absorption features are provided below. Figure 2 shows the red spectra in the period from January 14 to February 11, 2001. In the following sub-sections, we describe each of the individual spectra.

\subsection{January 14, 2001}

In Fig. 3, we present our first medium dispersion spectrum acquired on January 14, 2001. The ordinate axis is in units of intensity, but, as mentioned in Sect. 2, the errors in the flux calibrations should be large. Prominent emission lines clearly detected are of Fe II, C II, Mg II, Ti II, Cr II, [Fe II], Si II. Faint $\mathrm{He} \mathrm{I} 4471$ is also observed. The position of $\mathrm{H} \beta$ is indicated in the figure, but no prominent emission nor absorption is observed. These spectral features are consistent with those provided in the previous works (e.g., Wagner 2001; Kamath \& Anupama 2002) apart from the detection of [Fe II] lines, which is first reported in this work. The identifications and their equivalent widths are described in Table 2.

A rather broad absorption feature is seen at $4209 \AA$. This line was not seen in any other of our spectra (Fig. 1), nor in the spectrum obtained by Wagner (2001) on January 19, 2001. If this line had been due to Ca I 2, 4226.5, it should have been blueshifted by about $-1200 \mathrm{~km} \mathrm{~s}^{-1}$. Such a high velocity, however,

${ }^{2}$ http://physics.nist.gov/cgi-bin/AtData/lines-form was not found for the absorption component of $\mathrm{Ca}$ II $\mathrm{H}$ in the same spectrum. Also the P Cygni type absorption components of Fe II, Ti II, Cr II, and $\mathrm{Na}$ I which appeared in the later stages, do not have such a high velocity (Sect. 4.6).

The absorption component of $\mathrm{Ca}$ II $\mathrm{H}$ line was blue-shifted by $-310 \pm 40 \mathrm{~km} \mathrm{~s}^{-1}$ (Sect. 4.6). If we assume the same blueshift for this absorption line, its laboratory wavelength should be $4213.3 \pm 0.8 \AA$. We have identified this absorption line as the $\mathrm{CN}$ (cyanogen) molecular band at $4215 \AA$ A. Unfortunately, the spectral region of the other more intense $\mathrm{CN}$ band at $3883 \AA$ was not covered by our spectrum. Therefore, the identification is still rather questionable. We were, however, unable to find any other more probable candidate for this absorption.

Among all of the historical classical novae only DQ Her showed the CN molecular bands in its early decline stage (McLaughlin 1937; Sneden \& Lambert 1975, and references therein). The $\mathrm{CN}$ bands in DQ Her disappeared within a few days (McLaughlin 1937). Also the CN band of V445 Pup may have disappeared rapidly, because no other spectrum showed its trace.

Figure 4 shows a tracing of the red spectrum obtained on the same night. The position of $\mathrm{H} \alpha$ is indicated in the figure, but no emission nor absorption is seen. The strongest emission line in this region is found at $6590 \AA$. As seen later, high dispersion spectra showed that this emission line consisted of two peaks at 6584 and $6588 \AA$, which were identified as C II 2, 6578.0 and 6582.9 , respectively (Sect. 4.5). There may have been some contributions from C I 22, 6587.8 and Ca I 1, 6573 in this emission component. These results support the identifications by Wagner (2001). The other prominent emission lines were due to $\mathrm{Na} \mathrm{I}$, Fe II, Mg II, Si II, C I, C II, He I, and [Ti II]. The emission line of [Ti II] 9F, 6013.3 was not reported in the previous works. The identification of this line was made using also the high dispersion spectra (Sect. 4.4).

\subsection{February 2, 2001}

Three medium dispersion spectra were obtained on February 2, 2001, which covered the spectral range from 4000 to $7000 \AA$.

Figure 5 shows the blue spectrum covering the range from 4000 to $5200 \AA$. The $\mathrm{CN}$ absorption band had disappeared, while P Cygni type absorption components appeared on some Fe II lines. The emission lines in the range 4510-4580 , which were mainly due to Fe II multiplet numbers 37 and 38 (Moore 1959), weakened with respect to the earlier spectrum (Fig. 3), while the emission lines of C II, Mg II, and Si II roughly kept their equivalent widths. A weak emission is seen at $4865 \AA$. This feature, however, could not be identified as $\mathrm{H} \beta$ (see, Sect. 4.2).

Figure 6 shows the spectral range $4900-6000 \AA$. The prominent emission lines in this region are due to Fe II, [Fe II], Cr II, C II, C III, Na I, He I, and N II. The strongest emission line in this region is seen at $5900 \AA$, which was identified as Na I D1 and D2 blended with C II 5, 5890, 5892, and C III 20, 5894. We identify the lines, in addition, using a high dispersion spectrum obtained one day after the observations.

Figure 7 shows a tracing of the red spectrum. The emission lines of Fe II are weaker than measured in our earlier spectrum (Fig. 4). A P Cygni type absorption component is, in addition, detected for Fe II 74, 6456.

An emission line at $6550 \AA$ is observed to have increased slightly in intensity between the observations. This line could not be identified as [N II] 1F, 6548, because the other line of 
Table 2. Equivalent widths (-A) of prominent emission lines and some absorption lines measured on the medium dispersion spectra.

\begin{tabular}{|c|c|c|c|c|c|}
\hline W.L.(A) & Jan. 14 & Feb. 2 & Feb. 11 & Apr. 2 & Identifications \\
\hline 3921.5 & 7.3 & & & & C II 4, 19.0, 20.7 \\
\hline 3932.0 & -0.7 & & & & Ca II 1, 33.7 (abs) \\
\hline 3942.0 & 6.4 & & & & Fe II 3, 38.3; Fe II 190, 39.0 \\
\hline 3964.3 & -2.5 & & & & Ca II 1, 68.5 (abs) \\
\hline 3975.8 & 2.2 & & & & C II $37,72.4$ \\
\hline 3983.5 & 2.8 & & & & Fe II 3, 81.6 \\
\hline 4031.3 & 2.0 & 2.3 & & & Ti II 87, 28.3, 29.6 \\
\hline 4038.1 & 1.7 & 1.5 & & & Fe II 22, 35.5 \\
\hline 4079.5 & 1.1 & & & 2.4 & C II $36,76.0$ \\
\hline 4135.3 & 3.0 & 2.2 & 2.4 & & Cr II 26, 32.4; Si II 3, 30.9 \\
\hline 4150.7 & 2.1 & 1.7 & 2.0 & 1.0 & {$[\mathrm{Fe}$ II $] 21 \mathrm{~F}, 46.7 ; \mathrm{Fe}$ II $141,47.3$} \\
\hline 4165.5 & 0.9 & 0.5 & & 0.9 & C III 21,62.8;FeII 39,60.0;TiII 105,63.6 \\
\hline 4183.6 & 4.3 & 1.7 & 1.3 & & Fe II 28, 78.9; [Fe II] 23F, 79.0 \\
\hline 4192.0 & & 1.0 & & & [Fe II] 37F, 90.5; C III 18, 87.1 \\
\hline 4208.9 & -2.6 & & & & CN molecular band 15.0 (abs.) \\
\hline 4236.9 & 2.4 & 1.7 & 1.2 & & Fe II 27, 33.2; [Fe II] 21F, 31.6 \\
\hline 4250.6 & 2.0 & 1.5 & 2.2 & & C III 11, 47.6; [Fe II] 21F, 44.8; 36F, 49.1 \\
\hline 4272.7 & 14.3 & 21.5 & 14.7 & 22.7 & C II 6, 67.0,67.3; [Fe II] 36F, 70.0 \\
\hline 4291.9 & & & & 4.3 & {$[\mathrm{Fe}$ II] $7 \mathrm{~F}, 87.4$} \\
\hline 4305.3 & 1.2 & 1.2 & 0.5 & 1.8 & Fe II 27, 03.2 \\
\hline 4327.4 & 1.8 & 3.0 & 1.8 & 5.4 & C II 28, 22.0, 25.9; C III 7, 25.7 \\
\hline 4356.5 & 6.6 & 5.6 & 3.1 & 2.6 & Fe II 27, 51.8;[Fe II] 21F, 52.8; C I 15, 52.1 \\
\hline 4364.9 & & & & 2.6 & {$[\mathrm{Fe} \mathrm{II}]$ 7F, 59.3; [Fe II] 21F, 58.4} \\
\hline 4375.4 & 3.1 & 4.1 & 1.8 & 3.9 & C II 45, 71.6,72.5, 74.3; [Fe II] 21F, 72.4 \\
\hline 4388.0 & 1.8 & 3.1 & 1.6 & & Fe II 27, 85.4; [Fe II] 36F, 84.2 \\
\hline 4392.9 & & & & 6.4 & $\operatorname{Mg}$ II 10, 90.6 \\
\hline 4400.9 & 3.8 & 3.3 & 2.0 & & Ti II 51, 99.8, 94.1; Ti II 19, 95.0 \\
\hline 4421.6 & 4.3 & 4.3 & 2.9 & 3.9 & Fe II 27, 16.8; [Fe II] 6F, 16.3 \\
\hline 4453.0 & 2.6 & 3.0 & 2.0 & & {$[\mathrm{Fe}$ II $]$ 7F, 52.1; Fe II -, 51.5} \\
\hline 4471.2 & 2.0 & 1.8 & 1.1 & 2.3 & [Fe II] 6F, 70.3; He I 14, 71.6; Ti II 31, 68.5 \\
\hline 4483.9 & 4.7 & 4.6 & 4.0 & 5.9 & $\mathrm{Mg}$ II 4, 81.1,81.3 \\
\hline 4492.9 & 6.9 & 6.5 & 4.5 & 3.3 & Fe II 37, 89.2; [Fe II] 6F, 88.8 \\
\hline 4510.5 & 4.0 & 2.2 & 0.7 & 0.3 & Fe II 38, 08.3; [Fe II] 6F, 09.6 \\
\hline 4524.2 & 8.5 & 4.5 & 2.3 & 0.7 & Fe II 37, 20.2; Fe II 38, 22.6 \\
\hline 4536.4 & 4.2 & 1.3 & 0.8 & 0.9 & {$[\mathrm{Fe}$ II] 6F, 33.0; Fe II 37, 34.2} \\
\hline 4554.0 & & & & 0.5 & {$[\mathrm{Fe}$ II $] 6 \mathrm{~F}, 50.5,52.0 ; \mathrm{Fe}$ II $38,49.5$} \\
\hline 4560.3 & 5.8 & 2.1 & 1.0 & & Fe II 37, 55.9; Cr II 44, 55.0, 58.8 \\
\hline 4574.5 & 4.7 & 1.0 & 0.2 & & Cr II 16, 71.2; Ti II 82, 72.0 \\
\hline 4593.8 & 11.2 & 6.5 & 3.5 & 2.4 & Cr II 44, 88.2, 92.1; Fe II 38, 83.8 \\
\hline 4609.0 & & & & 1.5 & N II 5, 07.2 \\
\hline 4616.2 & & & & 3.3 & N II $5,13.9$ \\
\hline 4622.0 & 3.4 & 3.4 & 1.7 & 4.7 & Fe II 38, 20.5; C II 50, 18.9; Cr II 44, 18.8 \\
\hline 4631.8 & & & & 3.5 & C II 49, 30.5, 25.7 \\
\hline 4640.0 & 8.0 & 8.6 & 5.4 & 5.4 & Fe II 186, 35.3; Fe II 25, 34.6; C II-, 37.6, 38.9 \\
\hline 4649.4 & & & & 3.8 & C III 1, 47.4; O II 1, 41.8, 49.1 \\
\hline 4655.6 & & & & 5.0 & C III $1,50.2,51.4 ;$ O II $1,50.8$ \\
\hline 4662.6 & & 3.8 & & 6.3 & Fe II 43, 57.0; O II 1, 61.6; C II 5,63.5 \\
\hline 4673.6 & 3.7 & 5.3 & 4.7 & 6.3 & C III 5, 73.9; Fe II 25, 70.2; O II 1, 73.8 \\
\hline 4718.0 & 3.0 & 6.1 & 4.1 & 9.9 & [Fe II] 5F, 16.4; He I 13.2 \\
\hline 4733.5 & & & & 3.9 & C II 48, 27.2 \\
\hline 4744.0 & 9.9 & 14.3 & 9.0 & 3.8 & C II $1,38.1$ \\
\hline 4749.0 & & & & 5.7 & C II $1,45.0$ \\
\hline 4780.4 & 2.6 & 2.5 & 0.9 & & {$[\mathrm{Fe} \mathrm{II}] 20 \mathrm{~F}, 74.7$} \\
\hline 4806.2 & & 0.7 & & 1.2 & N II 20, 03.3; [Fe II] 4F, 99.3 \\
\hline 4811.7 & 0.7 & 0.7 & 0.9 & 1.1 & N II 20, 10.3; [Fe II] 20F, 14.6 \\
\hline 4832.6 & 2.3 & 1.3 & 0.5 & & Fe II 30, 25.7; C I 5, 26.7 \\
\hline 4866.1 & & 1.1 & 0.6 & 2.0 & C II -, 62.6; Cr II 30, 64.3; [Fe II] 20F, 52.7 \\
\hline 4912.4 & & 0.6 & 0.4 & & Ti II 114, 11.2; Cr II 190, 12.5 \\
\hline 4931.3 & 19.5 & 15.2 & 12.3 & 6.7 & Fe II 42, 23.9; C I 13, 32.0; He I 48, 21.9 \\
\hline 4955.7 & & 1.2 & & 3.4 & {$[\mathrm{Fe} \mathrm{II}] 20 \mathrm{~F}, 50.7$} \\
\hline 4972.5 & & 0.9 & 1.9 & 2.9 & {$[\mathrm{Fe}$ II $] 20 \mathrm{~F}, 73.4$} \\
\hline
\end{tabular}


Table 2. continued.

\begin{tabular}{|c|c|c|c|c|c|}
\hline W.L.(A) & Jan. 14 & Feb. 2 & Feb. 11 & Apr. 2 & Identifications \\
\hline 5002.0 & 2.1 & & 2.1 & & Fe II 25, 00.7; N II 19, 01.1 \\
\hline 5005.5 & & 1.1 & & 1.4 & {$[\mathrm{Fe}$ II $] 20 \mathrm{~F}, 05.5 ; \mathrm{N}$ II 19, 05.1} \\
\hline 5025.6 & 20.4 & 16.9 & 14.7 & 7.0 & Fe II 42, 18.4; [Fe II] 20F, 20.2; He I 4, 15.7 \\
\hline 5044.3 & 4.3 & 4.5 & 3.6 & 4.7 & Si II 5,41.1; C II 35, 44.8; C I 4, 41.7 \\
\hline 5058.5 & 7.9 & 6.6 & 6.3 & 6.2 & Si II 5, 56.0; C II 35, 47.2; C I 12, 52.1 \\
\hline 5112.2 & & 3.3 & 5.5 & 3.6 & [Fe II] 18F, 08.0; [Fe II] 19F, 11.6 \\
\hline 5121.9 & & & & 3.1 & C II 12, 21.7 \\
\hline 5153.7 & & 8.9 & 8.3 & 12.2 & C II 16, 51.1; Na I 8, 53.4 \\
\hline 5174.2 & & 10.0 & 9.5 & 4.0 & Fe II 42, 69.0; Mg I 2, 72.7 \\
\hline 5185.9 & & 2.1 & & 0.9 & [Fe II] $18 \mathrm{~F}, 82.0 ;[\mathrm{Fe}$ II] 19F, 84.8 \\
\hline 5202.7 & & 2.6 & 2.6 & 0.5 & Fe II 49, 97.6 \\
\hline 5240.5 & & 2.9 & & & Fe II 49, 34.6; Cr II 43, 37.3 \\
\hline 5263.5 & & 1.4 & & & [Fe II] 19F, 61.6; Ti II 70,62.1 \\
\hline 5277.2 & & 2.2 & & & {$[\mathrm{Fe}$ II $]$ 18F, 73.4; C III 4,73.4 } \\
\hline 5284.5 & & 3.4 & & & [Fe II] 35F, 83.1;Fe II 49,76.0; Cr II 43,80.1 \\
\hline 5305.6 & & 0.5 & & & [Fe II] 19F, 92.8; C III - 05.1 \\
\hline 5320.5 & & 5.6 & & & FeII 48, 16.8; FeII 49, 16.6; CrII 43, 14.0 \\
\hline 5335.3 & & 2.2 & & & [Fe II] 19F, 33.7; C II 11, 36.7 \\
\hline 5346.4 & & 4.8 & & & Fe II 48, 37.7 \\
\hline 5368.4 & & 4.6 & & & Fe II 48, 62.9; C II -, 63.6 \\
\hline 5383.7 & & 4.1 & & & [Fe II] 19F, 76.5; Ti II 69,81.0;C I 11,80.2 \\
\hline 5426.2 & & 1.0 & & & Fe II 49, 25.3; Ti II 80, 22.5 \\
\hline 5438.7 & & 0.9 & & & Fe II 48, 35.8; [Fe II] 18F, 33.2 \\
\hline 5537.5 & & 5.9 & & & N II 63, 35.4; C II 10, 36.0; Fe II 55,34.9 \\
\hline 5553.1 & & 3.2 & & & [Fe II] 39F, 51.3; N II 63, 52.0 \\
\hline 5647.4 & & 2.1 & & & [Fe II] $18 \mathrm{~F}, 44.0 ; \mathrm{C}$ II $15,48.1,40.5$ \\
\hline 5669.3 & & 9.5 & & & {$[\mathrm{Fe}$ II $]$ - 65.9; C III - 67.0; N II 3, 66.6} \\
\hline 5697.5 & & 2.6 & & & C III 2, 96.0; Ca I -, 88.5 \\
\hline 5713.1 & & 1.6 & & & N II 3, 10.8; Mg I 8, 11.1 \\
\hline 5780.9 & & -0.7 & & & Interstellar abs. \\
\hline 5804.7 & & 1.9 & & & Si II 8, 00.5, 06.8; C I 18, 93.5, 01.2 \\
\hline 5833.2 & & 3.4 & & & C III 22, 27.1; Fe II 58, 35.4 \\
\hline 5874.4 & 11.6 & 22.1 & 12.5 & & He I 11, 75.7; C III 20, 71.6; Si II 8, 68.4 \\
\hline 5905.3 & 31.1 & 43.3 & 25.4 & & NaI 1, 90.0,95.9; CII 5,90.0,91.7; CIII 20, 94.1 \\
\hline 5960.0 & 1.0 & & 1.8 & & Si II 4, 57.6; N II 28, 60.9 \\
\hline 5993.1 & 1.6 & & 0.7 & & Fe II 46, 91.4; Fe II 24, 86.5; Fe II 51, 90.6 \\
\hline 6019.8 & 2.2 & 1.7 & 1.7 & & [Ti II] 9F, 13.3 \\
\hline 6049.7 & & & 0.3 & & [Ti II] 9F, 47.5, 53.1 \\
\hline 6084.6 & & 3.5 & 1.9 & & Fe II 46, 84.1; Cr II 188, 81.5 \\
\hline 6104.8 & 4.1 & 7.4 & 7.1 & & C II 24,95.4, 98.6; [Ti II]9F, 96.0; Ca I 3,02.7 \\
\hline 6118.7 & 3.3 & 4.1 & & & Fe II 46, 13.3, 16.0;C II 19, 15.2; Ca I 3,22.2 \\
\hline 6165.1 & 8.7 & 10.9 & 7.0 & & Ca I 3, 62.6; C III 13,56.6 \\
\hline 6224.9 & & 1.2 & 0.5 & & Fe II 34, 19.5, 29.3 \\
\hline 6238.0 & & 0.9 & & & Fe II 74, 38.4; C II -, 35.5 \\
\hline 6254.9 & 12.0 & 8.8 & 5.0 & & Fe II 74, 47.6 \\
\hline 6295.3 & & 2.1 & & & unid. 6291.0 in the catalog of Meinel \\
\hline 6309.6 & 3.2 & 3.2 & 1.5 & & Fe II 200, 05.3; S II 19, 05.5 \\
\hline 6354.0 & 4.1 & 4.3 & 2.7 & & Si II 2, 47.1; N II 46, 47.1; Mg II 16,46.7 \\
\hline 6375.9 & 1.8 & 1.7 & 0.9 & & Si II 2, 71.4; Fe II 40, 69.5 \\
\hline 6385.0 & 1.1 & 1.7 & 0.6 & & Fe II -, 83.7; N II 2, 79.6 \\
\hline 6405.6 & 1.3 & 1.8 & 1.0 & & Ca I -, 95.2,05.9; Fe II 74, 07.3 \\
\hline 6420.6 & 2.6 & 2.7 & 1.4 & & Fe II 74, 16.9 \\
\hline 6437.3 & 4.2 & 3.9 & 1.5 & & Fe II 40, 32.7; Fe II 199, 33.9 \\
\hline 6465.6 & 14.6 & 10.2 & 7.2 & & Fe II 74, 56.4; C II -, 59.8 \\
\hline 6484.1 & & 3.3 & & & N II $8,82.1$ \\
\hline 6521.0 & 2.6 & 2.7 & 0.9 & & Fe II 40, 16.1; Fe II -, 17.0 \\
\hline 6550.4 & 3.1 & 4.9 & 5.8 & & $\mathrm{Mg}$ II 23, 45.8 \\
\hline 6560.5 & 1.6 & & & & [Fe II] 15F, 58.5; [Ti II] 8F, 48.9 \\
\hline 6592.0 & 59.9 & 65.3 & 42.9 & & C II 2, 78.0, 82.9; Ca I 1,72.8; [Ti II] 8F, 89.4 \\
\hline 6685.7 & 15.6 & 15.7 & 9.5 & & He I 46, 78.1; Fe II 210, 77.3; C I -, 79.6 \\
\hline 6727.3 & 3.5 & 4.2 & 2.2 & & [Ti II] 8F, 25.7,22.0; C II 21, 26.8 \\
\hline 6751.2 & 2.7 & 5.0 & 2.1 & & C II $21,38.4,50.2 ;$ C III $3,44.2$ \\
\hline 6808.8 & 13.0 & 22.3 & 9.6 & & C II 14, 91.3, 98.0,00.5; [Fe II] 31F, 09.2 \\
\hline 6831.9 & 5.4 & 3.0 & & & C I 21, 28.5; [Fe II] 31F, 29.0 \\
\hline
\end{tabular}


Table 3. Equivalent widths (-A) of prominent emission lines and some absorption lines measured on the high dispersion spetrum on February 3, 2001.

\begin{tabular}{|c|c|c|}
\hline W.L.(A) & Eqw(-A) & Identification \\
\hline 4343.0 & 1.1 & Ti II 32, 41.4; Ti II 20, 37.8; Fe II 32, 38.7 \\
\hline 4352.4 & 1.6 & Fe II 27, 51.8; [Fe II] 36F, 47.4 \\
\hline 4376.4 & 1.5 & C II 45, 72.5,74.3; Ti II 93, 74.9 \\
\hline 4386.9 & 1.3 & Fe II 27, 85.4; Fe II 32, 84.3; [Fe II] 6F, 82.8 \\
\hline 4397.7 & 0.5 & Ti II 51, 94.1; Ti II 19, 95.0 \\
\hline 4421.1 & 2.5 & Fe II 27, 16.8; [Fe II] 6F, 16.3; Ti II 51, 18.3 \\
\hline 4455.3 & 0.6 & {$[\mathrm{Fe}$ II $]$ 7F, 52.1; Ti II 19, 50.5} \\
\hline 4471.7 & 1.7 & Ti II 31, 68.5; [Fe II] 6F, 70.3; He I 14, 71.6 \\
\hline 4482.3 & 2.4 & {$[\mathrm{Fe}$ II $]$ 7F, 74.9; Fe II -, 80.7} \\
\hline 4485.5 & 2.3 & $\operatorname{Mg}$ II $4,81.1,81.3$ \\
\hline 4490.8 & 1.9 & Fe II 37, 89.2; [Fe II] 6F, 88.8 \\
\hline 4493.9 & 2.0 & Fe II 37, 91.4, [Fe II] 6F, 92.6 \\
\hline 4508.4 & 0.8 & Ti II 30, 06.7; Cr II 16, 04.5 \\
\hline 4513.7 & 1.0 & [Fe II] 6F, 09.6; Fe II 38, 08.3; N III 3, 10.5 \\
\hline 4524.3 & 3.4 & Fe II 37, 20.2; Fe II 38, 22.6 \\
\hline 4534.4 & 0.9 & Fe II 37, 34.2; N III 3, 30.8; [Fe II] 6F, 33.0 \\
\hline 4541.8 & 0.6 & Fe II 38, 41.5; Cr II 39, 39.6 \\
\hline 4549.3 & 0.7 & Fe II 38, 49.5; Cr II 16, 45.5; N III 3, 47.3 \\
\hline 4557.0 & 1.4 & Fe II 37, 55.9; Cr II 44, 55.0; [Fe II] 6F, 52.0,55.0 \\
\hline 4562.3 & 1.9 & $\mathrm{Cr}$ II $44,58.8 ; \mathrm{Fe}$ II $20,58.6$ \\
\hline 4574.6 & 0.8 & Cr II 16, 71.2; Ti II 82, 72.0 \\
\hline 4587.7 & 1.5 & Fe II 38, 83.8 \\
\hline 4594.2 & 1.7 & Cr II 44, 88.2, 92.1 \\
\hline 4621.4 & 2.2 & C II 50, 18.9; Cr II 44, 16.6,18.8; Fe II 38, 20.5 \\
\hline 4627.8 & 0.7 & C II 49, 25.7; Fe II 186, 25.9 \\
\hline 4634.1 & 1.6 & C II 49, 30.5; Fe II 37, 29.3 \\
\hline 4636.8 & 0.9 & Cr II 44, 34.1; [Fe II] 5F, 32.3; N III 2, 34.2 \\
\hline 4639.9 & 1.5 & C II -, 37.6; Fe II 186, 35.3; Ti II 38, 36.3 \\
\hline 4651.2 & 1.4 & Fe II 38, 48.2; C III 1, 47.4 \\
\hline 4660.8 & 1.0 & Fe II 43, 57.0; Fe II 170, 58.0 \\
\hline 4664.0 & 1.1 & Fe II 146, 60.9; Fe II 170, 58.0; Ti II 38, 62.7 \\
\hline 4670.3 & 1.0 & Fe II 37, 66.8; C II 5, 63.5; C III 5, 65.9 \\
\hline 4676.4 & 1.9 & Fe II 25, 70.2; [Fe II] 4F, 72.1; C III 5, 73.9 \\
\hline 4713.6 & 1.9 & He I $12,13.1,13.4 ;$ Fe II $26,13.2 ;$ N II $25,09.5$ \\
\hline 4719.7 & 2.8 & {$[\mathrm{Fe}$ II] $5 \mathrm{~F}, 16.4 ; \mathrm{N}$ II $68,18.4$} \\
\hline 4734.0 & 2.7 & {$[\mathrm{Fe}$ II $]$ 4F, 28.1; Fe II 43, 31.4; C II 48, 27.2} \\
\hline 4740.4 & 4.0 & C II $1,38.1 ;$ C II 48, 34.8; Mg II 18, 39.6 \\
\hline 4746.5 & 4.0 & C II 1, 45.0; [Fe II] 20F, 45.5 \\
\hline 4753.1 & 3.5 & {$[\mathrm{Fe} \mathrm{II}]$ 5F, 50.6; Na I 11, 47.9,51.8 } \\
\hline 4762.9 & 1.4 & Cr II 176, 61.4; Fe II 169, 60.2; C I 6, 62.4 \\
\hline 4772.7 & 0.6 & C I 6, 66.6, 71.7 \\
\hline 4778.0 & 1.6 & [Fe II] 20F, 74.7; N II 20, 74.2 \\
\hline 4784.5 & 1.2 & N II 20, 79.7, 81.2; Ti II 92, 80.0 \\
\hline 4805.0 & 0.7 & N II 20, 03.3; [Fe II] 4F, 98.3, 99.3; Ti II 92, 05.1 \\
\hline 4839.4 & 1.6 & Cr II 30, 36.2; [Ti II] 10F, 37.4 \\
\hline 4848.5 & 1.5 & Fe II 25, 46.5; [Fe II] 3F, 43.5 \\
\hline 4855.4 & 2.1 & [Fe II] 20F, 52.7; Cr II 30, 48.2 \\
\hline 4865.6 & 2.9 & C II -, 62.6; Cr II 30, 64.3 \\
\hline 4913.6 & 1.2 & Ti II 114, 11.2; [Fe II] 20F, 05.4 \\
\hline 4926.7 & 7.7 & Fe II 42, 23.9; He I 48, 21.9 \\
\hline 5006.9 & 1.3 & N II 19, 01.5,05.1; [Fe II] 20F, 05.5 \\
\hline 5021.5 & 8.5 & Fe II 42, 18.4; [Fe II] 20F, 20.2; He I 4, 15.7 \\
\hline 5043.2 & 2.7 & Si II 5, 41.1; C II 35, 44.8; [Fe II] 20F, 43.5; C I 4, 41.7 \\
\hline 5056.7 & 4.2 & Si II 5, 56.0; C II 35, 47.2; [Fe II] 20F, 49.3 \\
\hline 5102.6 & 1.7 & Fe II 35, 00.7; Fe II 185, 00.8 \\
\hline 5112.5 & 2.3 & {$[\mathrm{Fe} \mathrm{II}] 18 \mathrm{~F}, 08.0 ;[\mathrm{Fe} \mathrm{II}]$ 19F, 11.6} \\
\hline 5146.9 & 1.8 & C II $16,43.5,45.2$ \\
\hline 5156.7 & 5.8 & C II $16,51.1 ; \mathrm{Na}$ I $8,53.4 ;$ Cr II $24,53.5$ \\
\hline 5173.0 & 10.1 & $\mathrm{Fe}$ II $42,69.0$ \\
\hline 5187.6 & 2.9 & {$[\mathrm{Fe} \mathrm{II}]$ 19F, 84.8; [Fe II] 18F, 82.0} \\
\hline 5200.5 & 3.8 & $\mathrm{Fe}$ II 49, 97.6 \\
\hline 5239.1 & 1.7 & Fe II 49, 34.6 \\
\hline 5265.2 & 1.1 & [Fe II] 19F, 61.6; Ti II 70, 62.1 \\
\hline 5276.9 & 1.6 & [Fe II] 18 F, 73.4; Fe II 185, 72.4; C III 4, 72.6 \\
\hline 5283.4 & 2.6 & Fe II 49, 76.0; Cr II 43, 79.9, 80.1 \\
\hline
\end{tabular}


Table 3. continued.

\begin{tabular}{|c|c|c|}
\hline$\overline{\text { W.L.(A) }}$ & $\overline{\mathrm{Eqw}(-\mathrm{A})}$ & Identification \\
\hline 5306.5 & 1.8 & [Fe II] 19F, 92.8; C III -, 05.1 \\
\hline 5317.5 & 3.4 & Cr II 43, 13.6; N II 69, 13.4 \\
\hline 5322.6 & 3.1 & Fe II 48, 16.8; Fe II 49, 16.6; N II 69, 21.0 \\
\hline 5337.5 & 2.5 & [Fe II] 19F, 33.7; C II 11, 36.7; Cr II 43, 34.9 \\
\hline 5345.6 & 2.3 & Fe II 48, 37.7; N II 69, 40.2 \\
\hline 5352.1 & 2.6 & Fe II 49, 46.6; [Fe II] 18F, 47.7; N II 69, 51.2 \\
\hline 5368.5 & 2.6 & Fe II 48, 62.9; C II -, 63.6 \\
\hline 5383.9 & 2.2 & [Fe II] 19F, 76.5; Ti II 69, 81.0 \\
\hline 5425.3 & 1.1 & Cr II 23, 20.9; Ti II 80, 22.5 \\
\hline 5430.8 & 1.4 & Fe II $49,25.3$ \\
\hline 5439.5 & 1.5 & Fe II 48, 35.8; [Fe II] 18F, 33.2 \\
\hline 5526.3 & 0.9 & [Fe II] 33F, 23.3; Fe II 56, 25.1 \\
\hline 5537.9 & 3.8 & Fe II 55, 34.9; Fe II 48, 35.8; N II 63, 35.4 \\
\hline 5553.8 & 2.4 & [Fe II] 39F, 51.3; N II 63, 52.0 \\
\hline 5643.4 & 0.7 & C II 15, 40.5; Si II 9, 39.5 \\
\hline 5649.4 & 1.0 & [Fe II] 18F, 44.0; C II 15, 48.1 \\
\hline 5659.3 & 0.9 & [Fe II] $17 \mathrm{~F}, 54.9 ; \mathrm{Fe}$ II 57, 57.9 \\
\hline 5665.0 & 2.1 & Cr II 34, 62.6; [Fe II] 33F, 59.8; C II 15, 62.5 \\
\hline 5670.4 & 2.3 & {$[\mathrm{Fe}$ II $]-, 65.9 ; \mathrm{C}$ III -, 67.0; N II 3, 66.6} \\
\hline 5685.1 & 3.4 & Ca I -, 82.9; N II 3, 79.6; [Fe II] 33F, 83.6 \\
\hline 5693.2 & 1.2 & Ca I-, 88.5; Na I 6, 88.2 \\
\hline 5697.1 & 1.3 & Fe II 47, 91.4; Ti II 79, 92.0 \\
\hline 5701.2 & 1.9 & C III 2, 96.0; Fe II 18, 96.1 \\
\hline 5712.1 & 3.0 & N II 3, 10.8; Mg I 8, 11.1 \\
\hline 5723.8 & 1.5 & [Fe II] 33F, 21.4 \\
\hline 5768.0 & 1.0 & Ca I -, 64.3 \\
\hline 5781.6 & -0.58 & Interstellar abs. \\
\hline 5875.6 & 20.2 & He I 11, 75.6; C III 20, 71.6; Ca I 47, 57.5 \\
\hline 5890.7 & $\ldots$ & Interstellar abs. Na I 90.0 low velocity \\
\hline 5891.8 & $\ldots$ & Interstellar abs. Na I 90.0 high velocity \\
\hline 5896.7 & -0.34 & Interstellar abs. Na I 95.9 low velocity \\
\hline 5897.8 & -0.62 & Interstellar abs. Na I 95.9 high velocity \\
\hline 5901.5 & 28.8 & Na I 1, 90.0, 95.9; C II 5, 90.0, 91.7; C III 20, 94.1 \\
\hline 5993.0 & 1.9 & Fe II 46, 91.4; Fe II 24, 86.5; Fe II 51, 90.6 \\
\hline 6018.6 & 2.9 & [Ti II] 9F, 13.3 \\
\hline 6051.5 & 1.4 & [Ti II] 9F, 47.5, 53.1 \\
\hline 6075.2 & 0.7 & Cr II 193, 68.0,69.7; Cr II 105, 70.1 \\
\hline 6085.0 & 2.9 & Cr II 188, 81.5; Fe II 46, 84.1 \\
\hline 6102.8 & 5.4 & C II 24, 95.4,98.6; [Ti II] 9F, 96.0; Ca I 3, 02.7 \\
\hline 6115.0 & 2.0 & Fe II 46, 13.3; C II 19, 15.2 \\
\hline 6150.7 & 1.9 & Fe II $74,47.7,49.2$ \\
\hline 6159.5 & 3.1 & C III 13, 54.4, 56.6; Fe II 161, 55.2 \\
\hline 6167.1 & 4.4 & Ca I 3, 62.2; Fe II 161, 60.8 \\
\hline 6237.6 & 1.2 & Fe II -, 33.5; C II -, 35.5 \\
\hline 6250.9 & 5.4 & Fe II 74, 47.6, 38.4 \\
\hline 6352.6 & 4.1 & Si II 2, 47.1; Mg II 16, 46.7 \\
\hline 6374.7 & 2.0 & Si II 2, 71.4; Fe II 40, 69.5 \\
\hline 6386.0 & 1.6 & Fe II-, 83.7; N II 2, 79.6 \\
\hline 6405.5 & 1.3 & Ca I -, 95.2, 05.9 \\
\hline 6419.9 & 1.7 & Fe II 74, 16.9 \\
\hline 6435.6 & 1.5 & Fe II 40, 32.7 \\
\hline 6462.3 & 5.0 & Fe II 74, 56.4; C II -, 59.8 \\
\hline 6520.7 & 0.8 & Fe II 40, 16.1; Fe II -, 17.0 \\
\hline 6550.3 & 3.1 & $\mathrm{Mg}$ II 23, 45.8 \\
\hline 6560.5 & 1.8 & [Fe II] 15F, 58.5; [Ti II] 8F, 48.9 \\
\hline 6578.8 & 4.6 & Ca I $1,72.8$ \\
\hline 6583.9 & 11.2 & C II $2,78.0$ \\
\hline 6590.2 & 15.1 & C II 2, 82.9; C I 22, 87.8 \\
\hline 6682.5 & 6.9 & He I 46, 78.1; C I -, 79.6, etc.; Ti II 112, 80.3 \\
\hline 6726.5 & 1.5 & C II 21, 26.8; [Ti II] 8F, 22.0, 25.7; Ti II 112, 17.9 \\
\hline 6743.5 & 1.9 & C II $21,38.4,33.6,42.1$ \\
\hline 6753.3 & 1.7 & C II 21, 50.2, 54.8; C III 3, 44.2 \\
\hline 6786.9 & 1.8 & C II $14,83.8,79.7,80.3$ \\
\hline 6793.7 & 2.4 & C II $14,87.1,91.3$ \\
\hline 6804.8 & 6.0 & C II $14,98.0,00.5$ \\
\hline 6815.7 & 4.9 & C II 14, 12.2; [Fe II] 31F, 09.2 \\
\hline 6831.4 & 3.0 & C I 21, 28.5; [Fe II] 31F, 29.0 \\
\hline
\end{tabular}




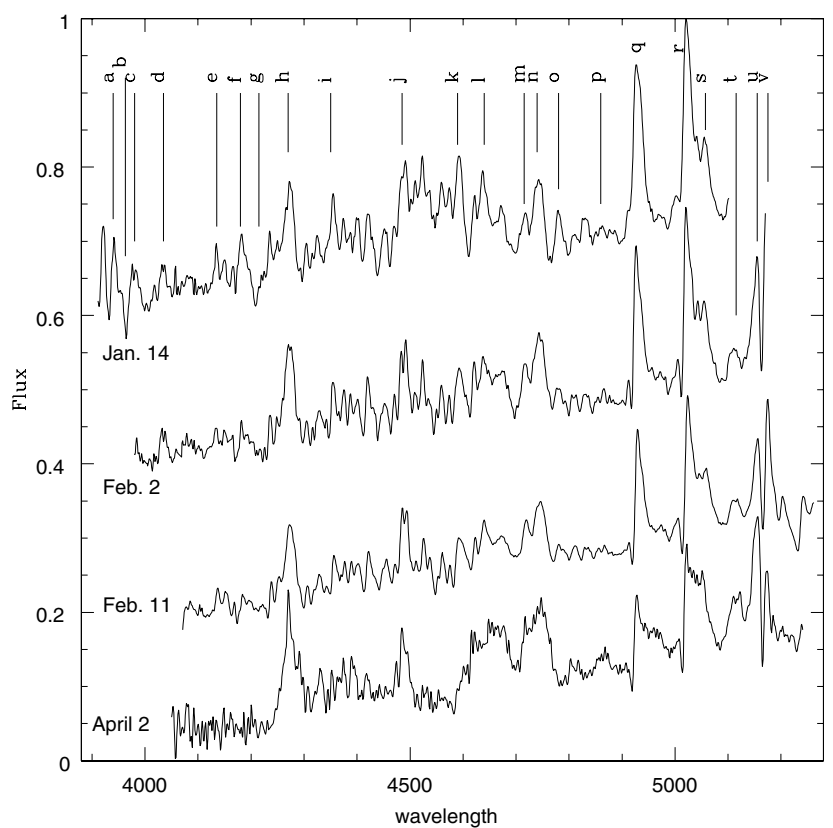

Fig. 1. Spectral evolution of V445 Pup in the blue region. a: Fe II 3, 3938; Fe II 190, 3939; b: Ca II 1, 3968 (abs.); c: C II 37, 3972; Fe II 3, 3982; d: Ti II 87, 4028,4030; Fe II 22, 4035; e: Cr II 26, 4132; Si II 3, 4131; f: Fe II 28, 4179; [Fe II] 23F, 4179; g: CN (cyanogen) absorption band 4215; h: C II 6, 4267; [Fe II] 36F, 4270; i: Fe II 27, 4352; [Fe II] 21F, 4353; j: Mg II 4, 4481; Fe II 37, 4489; [Fe II] 6F, 4489; k: Fe II 38, 4584; Cr II 44, 4588,4592; 1: Fe II 186, 4635; Fe II 25, 4635; C II -, 4638,4639; m: He I 12, 4713; [Fe II] 5F, 4716; n: C II 1, 4738,4745; o: [Fe II] 20F, 4775; N II 20, 4774,4780,4781; p: expected position of $\mathrm{H} \beta$; q: Fe II 42, 4924; He I 48, 4922; r: Fe II 42, 5018; He I 4, 5016; s: Si II 5, 5056; C II 35, 5047; t: [Fe II] 18F, 5108; [Fe II] 19F, 5112; u: C II 16, 5151; Na I 8, 5153; v: Fe II 42, 5169; Mg I 2, 5173.

[N II] at $5755 \AA$, which usually emerges prior to that of [N II] $1 F, 6548$ in novae spectra, was not detected. There were two possible identifications of this line: $\mathrm{Mg}$ II 23, 6545.8 and [Ti II] 8F, 6548.9. We have rejected the latter, because its intensity relative to that of [Ti II] 9F, 6013.3 largely varied as seen in Fig. 2.

\subsection{February 11, 2001}

Two spectra in the blue, 4100-5250 , and red, 5850-7000 A, regions were obtained on February 11, 2001. Their tracings are shown in Figs. 8 and 9. The overall spectral features were roughly the same as that on February 2 (Figs. 5, 7). The P Cygni type absorption components of Fe II lines developed, while their emission components continued to fade. The emission line of Mg II 4, 4481 was stronger than that of Fe II 37, 4489, while the growth of the emission line of Mg II 23, 6545.8 was notable in the red region.

\subsection{April 2, 2001}

Our last spectrum, which acquired data for primarily blue wavelengths, was obtained on April 2, 2001. Its tracing is shown in Fig. 10. As seen in the Figs. 1 and 10, the spectral features largely changed after the earlier observations. Some emission lines of Fe II were observed, but weakened. The prominent emission lines were of C II 6, 4267, Mg II 4, 4481, C II 16, 5151, and so on. The emission lines of [Fe II] strengthened relative to the

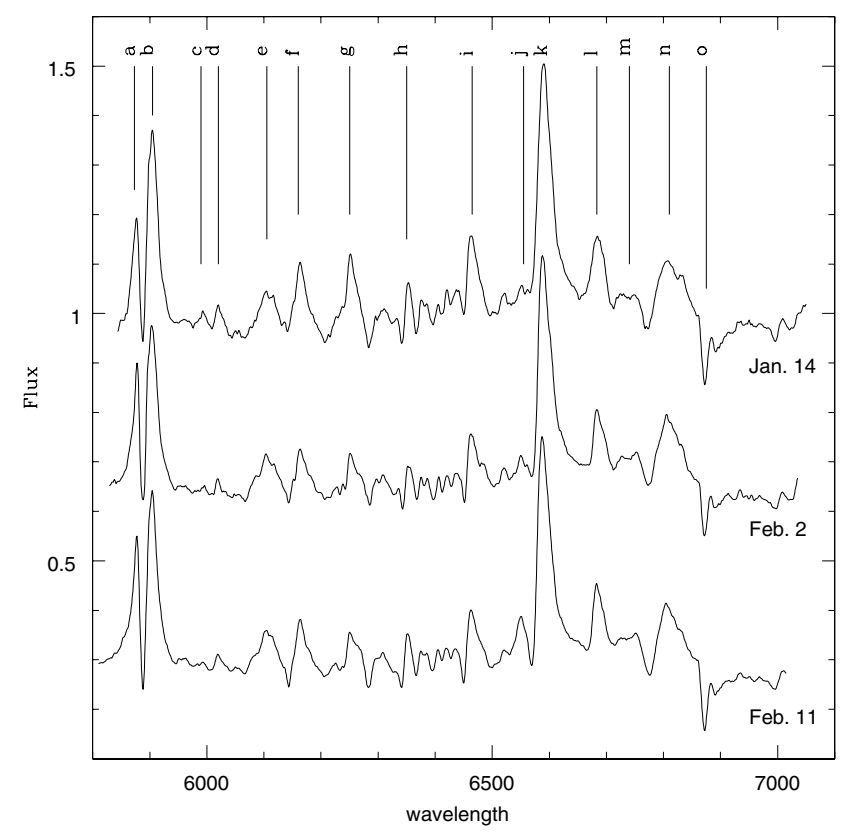

Fig. 2. Spectral evolution of V445 Pup in the red region. a: He I 11, 5876; C III 20, 5872; Si II 8, 5868; b: Na I 1, 5890,5896; C II 5, 5890, 5892; c: Fe II 46, 5991; Fe II 24, 5986; Fe II 51, 5991; d: [Ti II] 9F, 6013; e: C II 24, 6095,6099; [Ti II] 9F, 6096; Fe II 46, 6113; Ca I 3, 6122 etc.; f: Ca I 3, 6163; C III 13, 6157; g: Fe II 74, 6248; h: Si II 2, 6347; N II 46, 6347; Mg II 16, 6347; i: Fe II 74, 6456; C II -, 6460; j: Mg II 23, 6546; k: C II 2, 6578, 6583; Ca I 1, 6573; 1: He I 46, 6678; Fe II 210, 6677; C I -, 6680 etc.; m: C II 21, 6727, 6734, 6738 etc.; n: C II 14, 6798, 6801, 6812 etc.; o: Atmospheric $B$ band.

Fe II lines. Two broad emission bands are seen at $4650 \pm 40$ and $4740 \pm 30 \AA$ A . As discussed in Sect. 4.1 with the high dispersion spectrum, these bands may have consisted of the emission lines of C II and C III. The emission lines of He I at 4471 and 4713 were seen, but no trace of He II 4686 was detected.

These spectral properties suggest that this object was still in the early decline stage on April 2, 2001, which is consistent with the very slow decline rate in the light curve.

\section{High dispersion spectra}

High dispersion spectra were obtained on February 3 and 5, 2001. Since both spectra are almost identical, we report on the spectrum acquired on February 3. The overall profile of the high dispersion spectrum is shown in Fig. 11. In the following five subsections, we discuss this spectrum in detail.

\subsection{Spectral region $4340-4800 \AA$}

Figure 12 shows the blue region of the high dispersion spectrum. Since the signal-to-noise ratio of the spectrum was low, the tracing is smoothed over every five pixels. The effective spectral resolution of the figure is $\lambda / \Delta \lambda \cong 4000$.

The narrow absorption lines in this region are identified to be Fe II 20, 4559; Fe II 27, 4352, 4385, 4417; Fe II 37, 4456, 4629; Fe II 38, 4523, 4550, 4584, 4620; Ti II 19, 4395, 4444, 4450; Ti II 51, 4394, 4400; Cr II 44, 4559, 4634; and Mg II 4, 4481. Most of them have emission components. The emission lines of [Fe II] 6F, 4452, 4470, 4510, 4533; [Fe II] 7F, 4452; and He I 4471, are also detected. The broad emission band at 


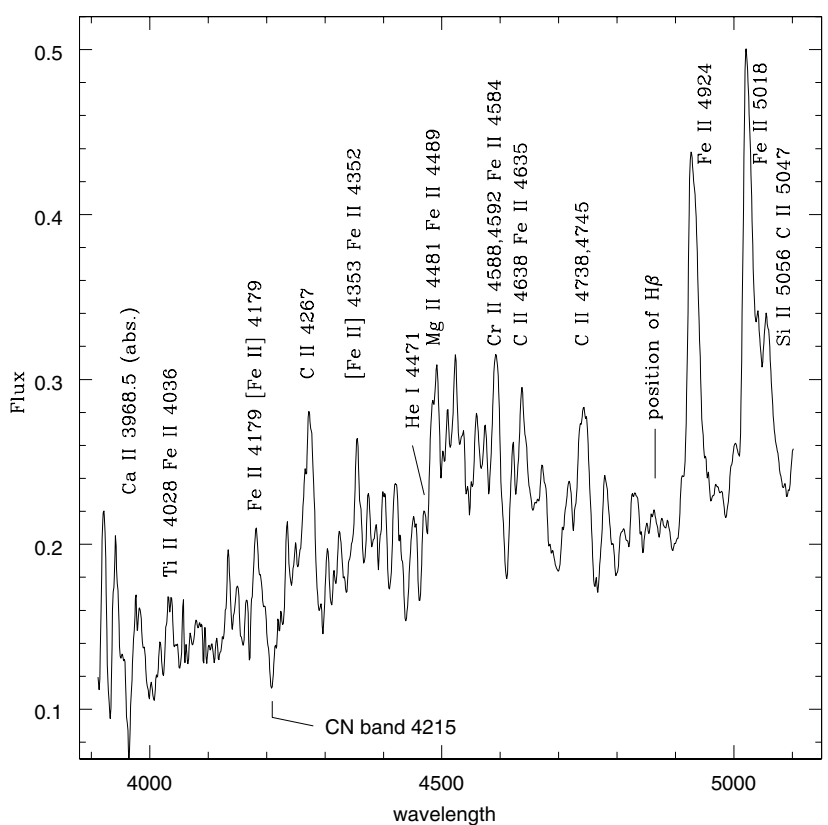

Fig. 3. A spectrum of V445 Pup on Jan. 14, 2001. The ordinate is intensity scale.

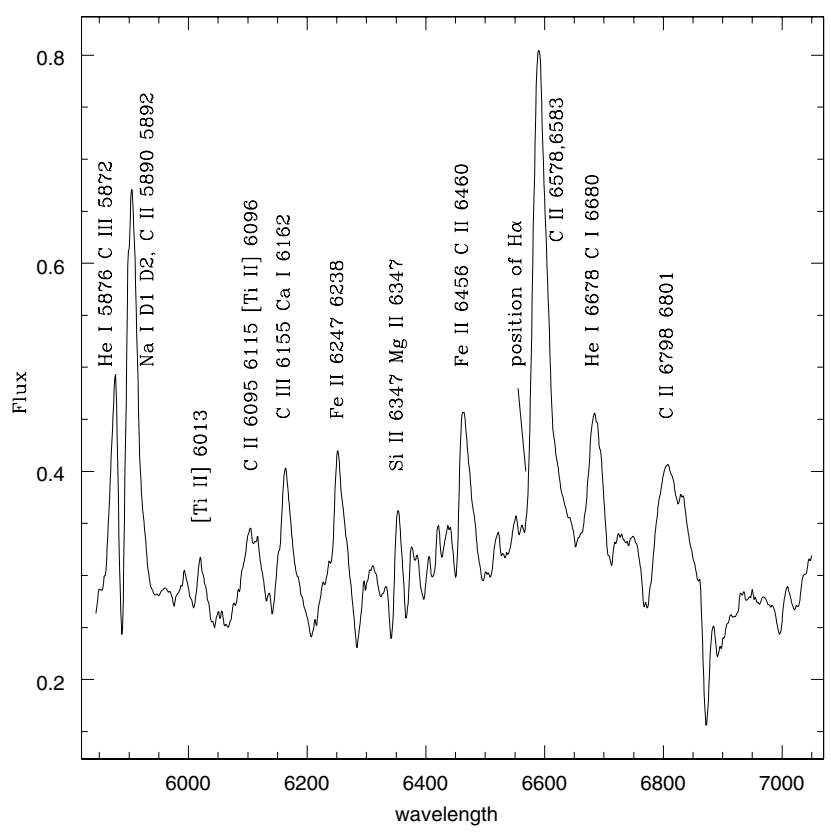

Fig. 4. A spectrum of V445 Pup on Jan. 14, 2001. The ordinate is intensity scale.

$4650 \pm 30 \AA$ may be due to C II 49, 4631; C III 1, 4647, 4650, 4651; C III 3, 4664, 4666, and 4674. There are probably also some contributions from N II and N III lines. The broad emission band at $4740 \pm 30 \AA$ in Fig. 10 (Sect. 3.4) is decomposed into two bands at 4715 and 4745 . The former may be due to He I 4713 and [Fe II] 5F, 4716, while the latter could be a blend of C II 1, 4738, 4745; C II 48, 4727, 4735; Mg II 18, 4740; and [Fe II] 20F, 4745 .

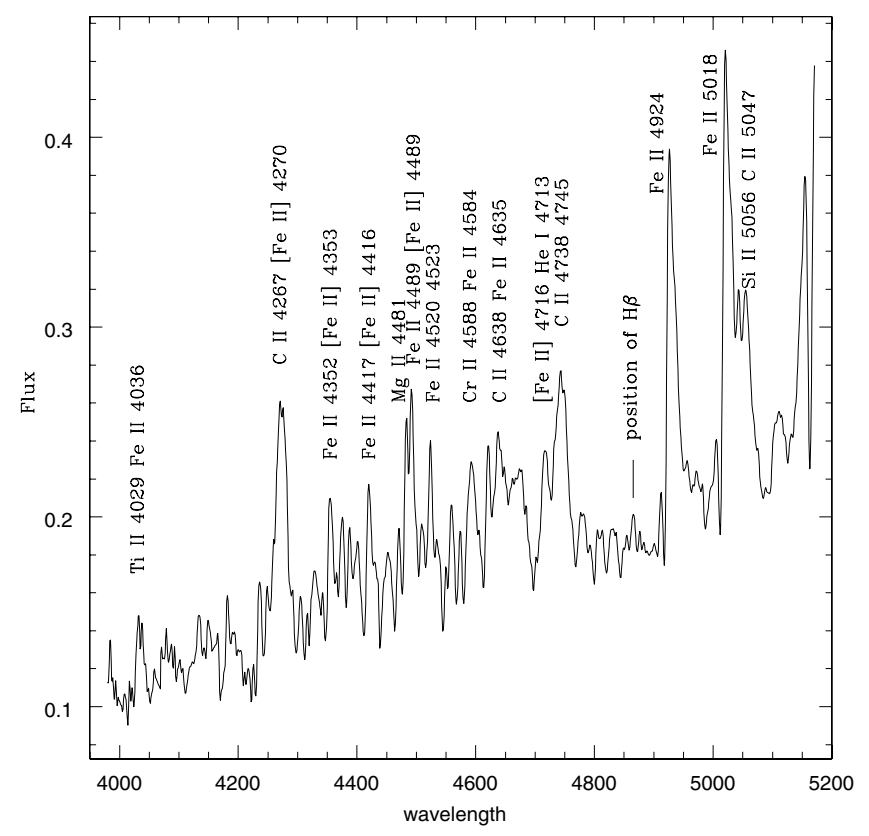

Fig. 5. A spectrum of V445 Pup on Feb. 2, 2001. The ordinate is intensity scale.

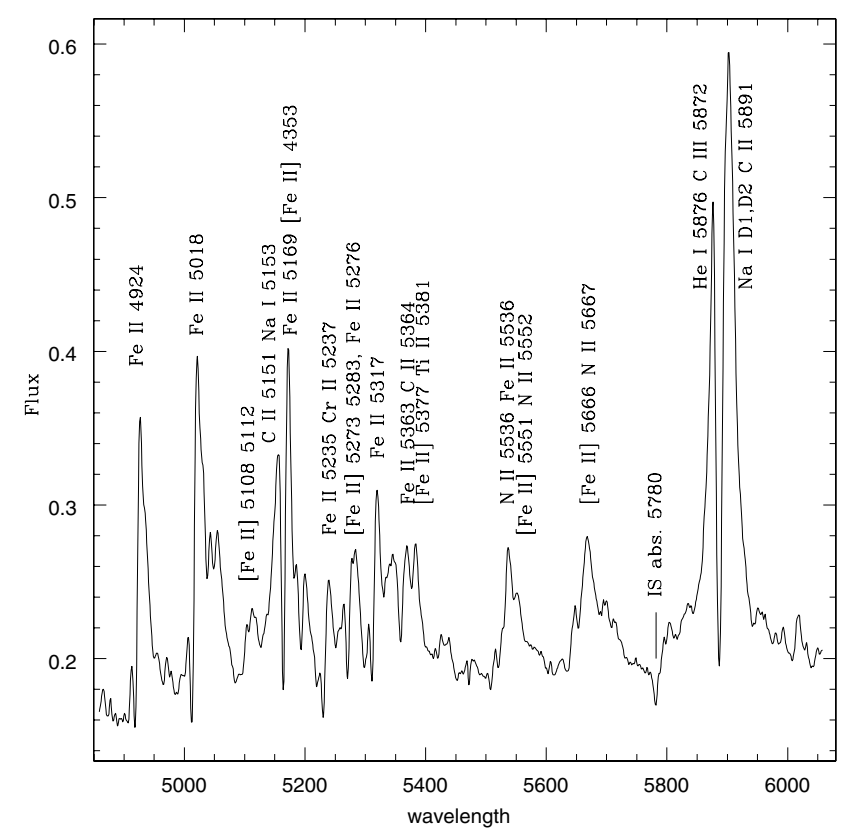

Fig. 6. A spectrum of V445 Pup on Feb. 2, 2001. The ordinate is intensity scale.

\subsection{Spectral region $4800-5300 \AA$}

Figure 13 shows the spectral region $4800-5300 \AA$ A. The emission lines with P Cygni type absorption components of Fe II 42, 4924, 5018, and 5169 are dominant in this region. There may have been contributions from the emissions of He I 4922 and 5016, but their absorption components were not detected. The emission lines of Si II 5, 5041 and 5056 are seen, but the former may have been blended with C I 4, 5039, 5042; C II 35, 5045; and [Fe II] 20F, 5043, while the latter may have been blended with 


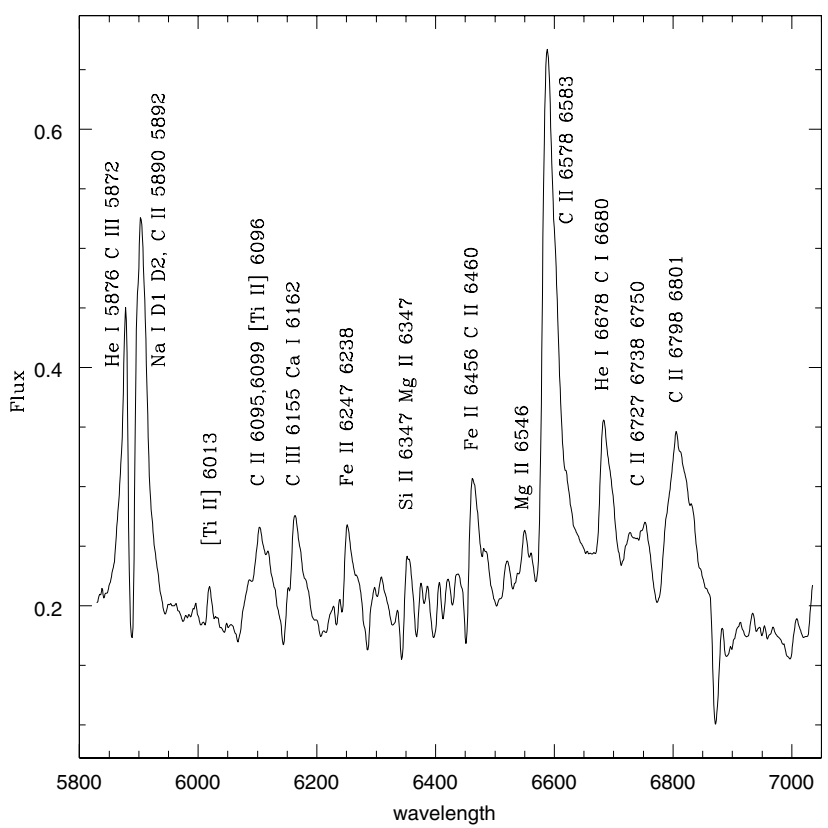

Fig. 7. A spectrum of V445 Pup on Feb. 2, 2001. The ordinate is intensity scale.

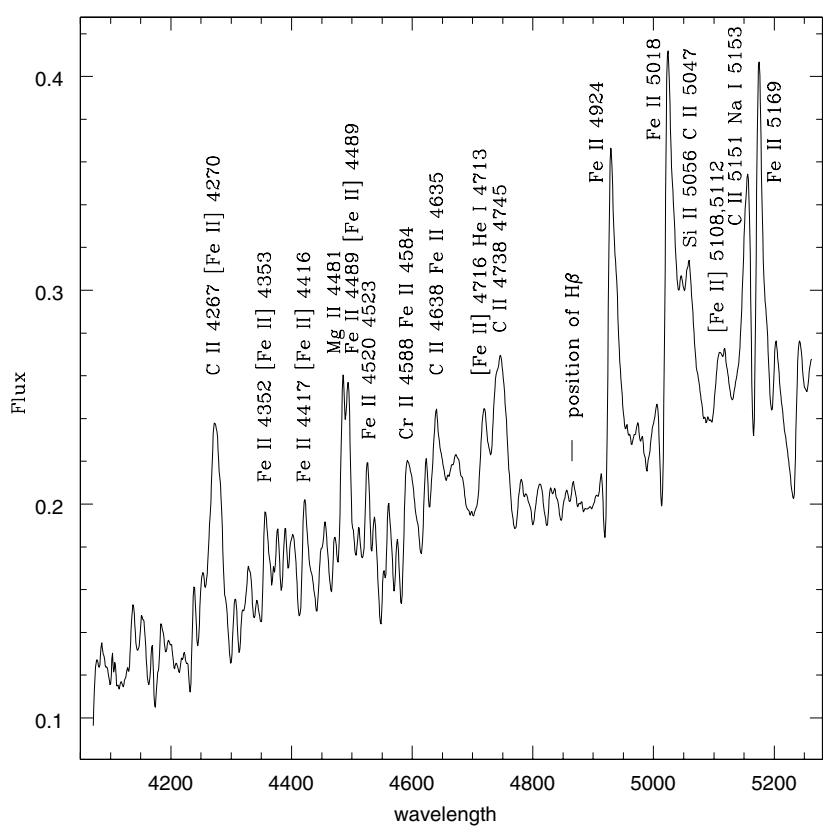

Fig. 8. A spectrum of V445 Pup on Feb. 11, 2001. The ordinate is intensity scale.

C II 35, 5047; C I 12, 5052; and [Fe II] 20F, 5049. The emission line at $5157 \AA$ has been identified as a blend of C II 16, 5151 and $\mathrm{Na}$ I 8, 5153.

A weak emission hump was observed about $4865 \AA$, which increased in intensity after this observation as seen in Fig. 10. The equivalent width of this emission was $-2.9 \AA$ (Table 3 ). If this emission had been due to $\mathrm{H} \beta$, there would have been an emission of $\mathrm{H} \alpha$ with an equivalent width of about $-5 \AA$ in the same spectrum. Such an emission, however, was not detected

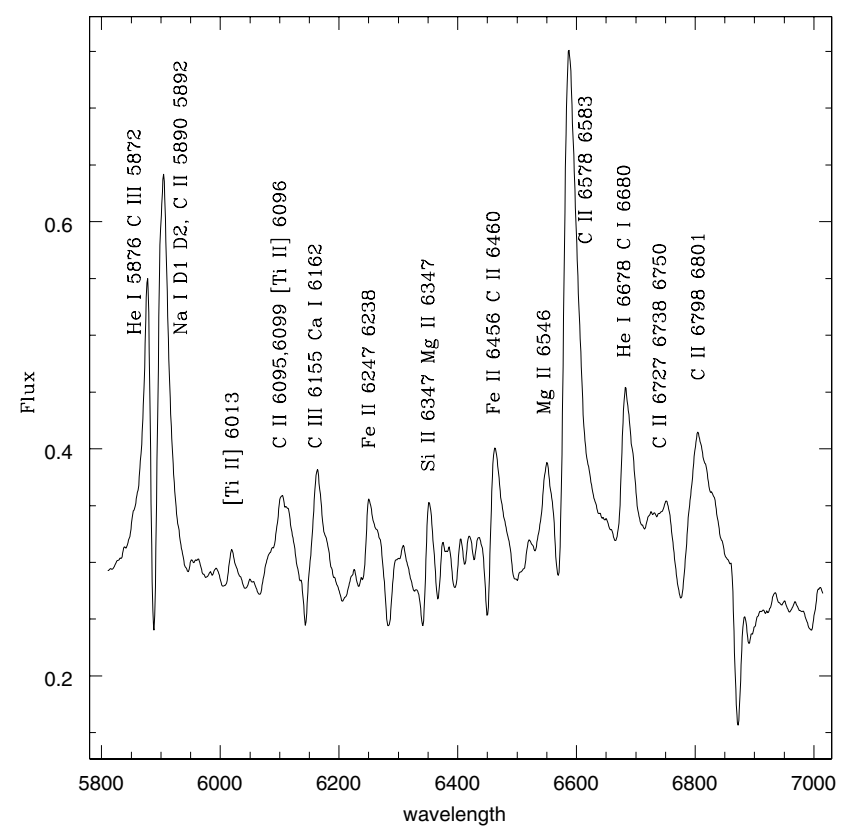

Fig. 9. A spectrum of V445 Pup on Feb. 11, 2001. The ordinate is intensity scale.

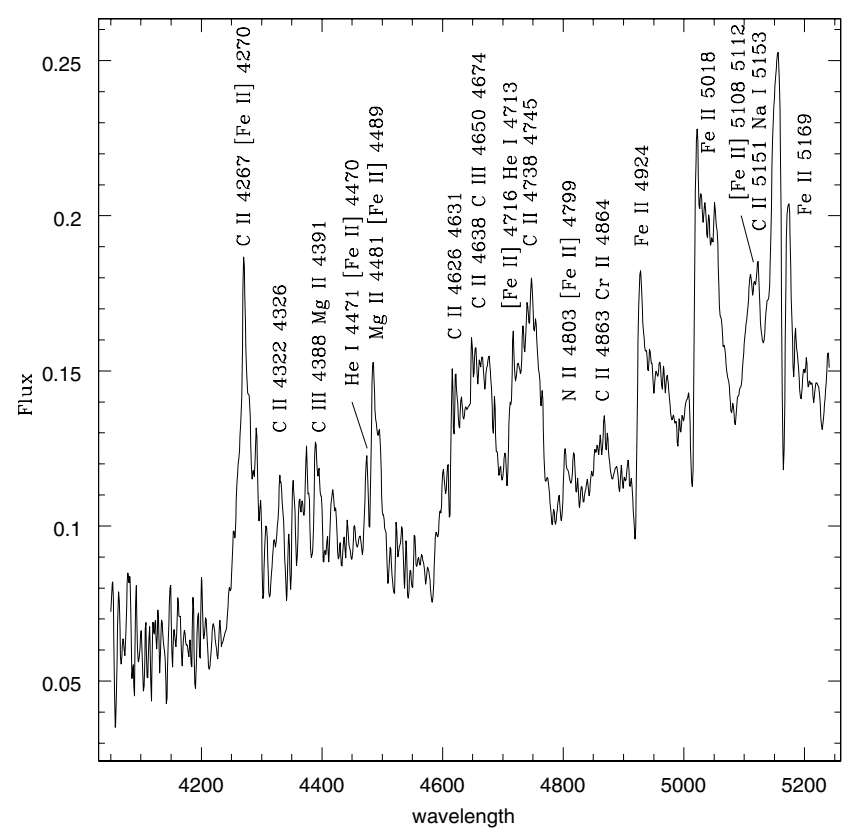

Fig. 10. A spectrum of V445 Pup on April 2, 2001. The ordinate is intensity scale.

(Sect. 4.5). We estimated an upper limit for the equivalent width of the emission of $\mathrm{H} \alpha$ at $-0.2 \AA$ (Sect. 4.5). Thus, the upper limit for the equivalent width of the $\mathrm{H} \beta$ emission is about $-0.1 \AA$, which means that $\mathrm{H} \beta$ could not be a main contributor to the emission hump at $4865 \AA$. This emission hump may have been due to C II lines of unknown multiplet number at 4862.6 and 4867.1 (Meinel et al. 1968); Cr II 30, 4864; and [Fe II] 20F, 4852.7. The narrow absorption lines in the region 5180-5300 $\AA$ are of Fe II 49, 5198, 5235, 5276; and Ti II 70, 5226.5. 


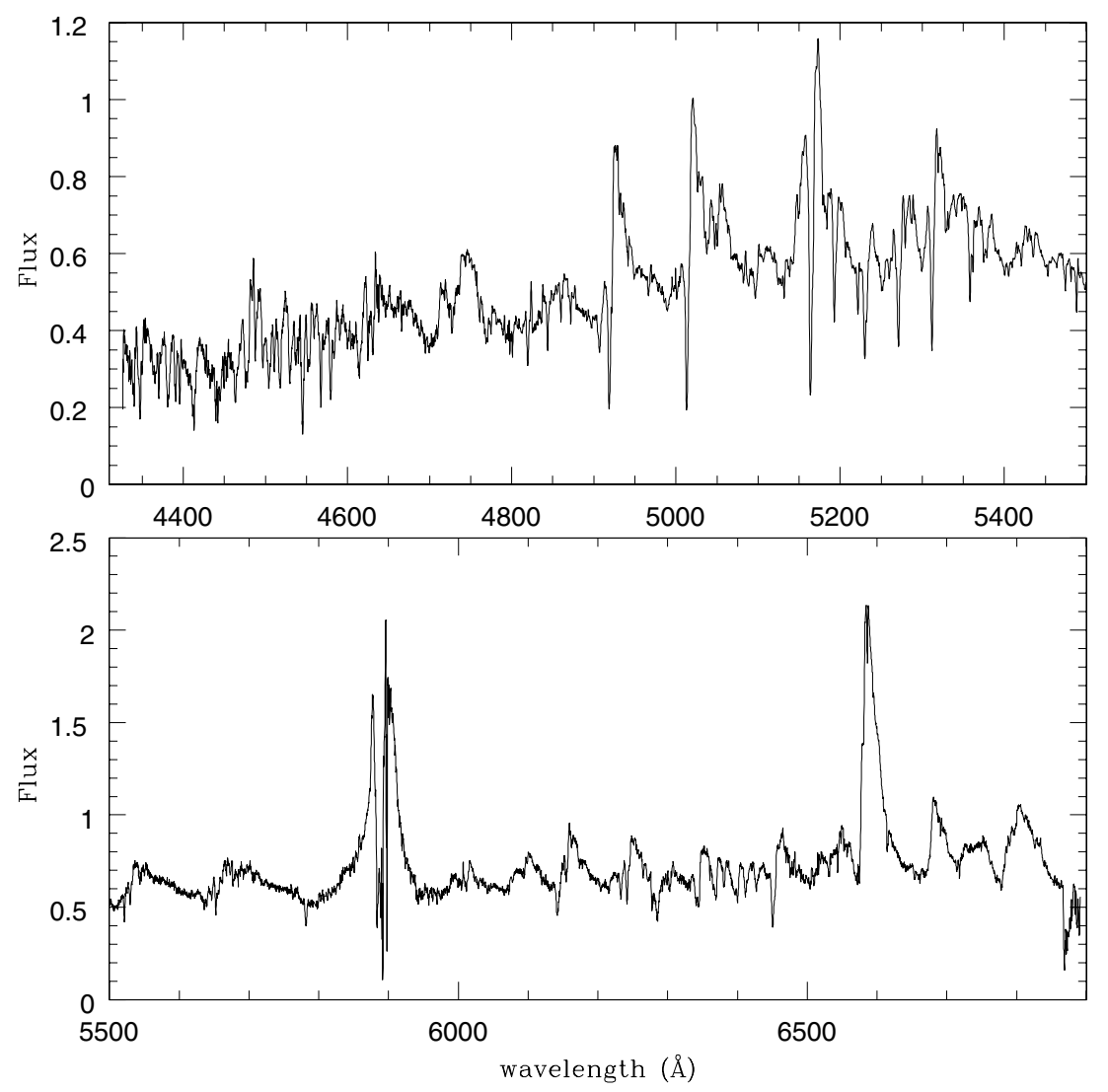

Fig. 11. A high dispersion spectrum of V445 Pup on February 3, 2001. The ordinate is intensity scale.

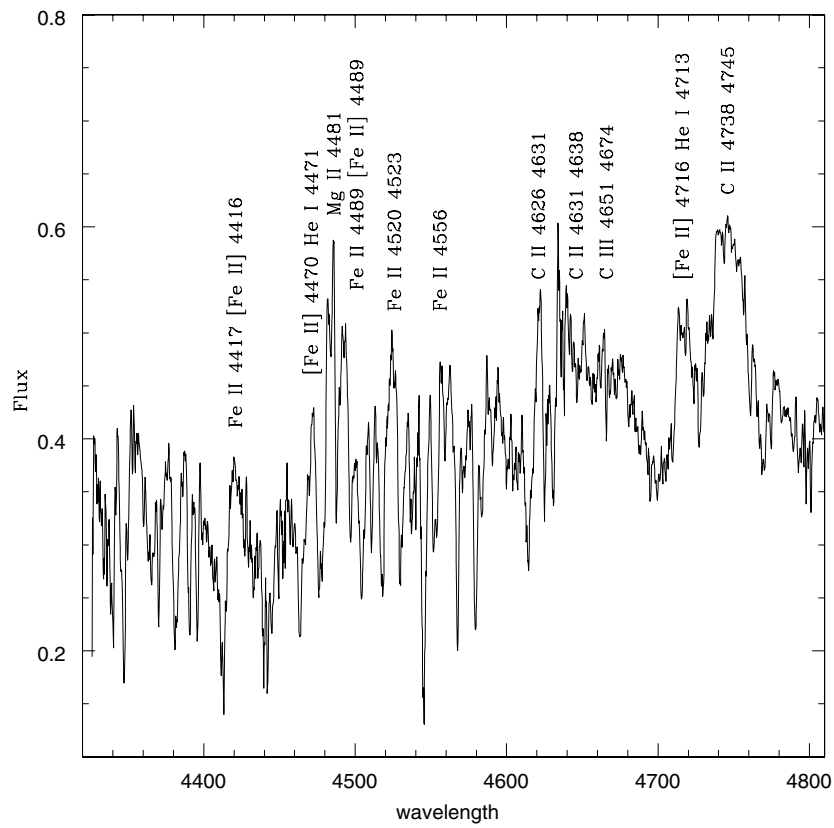

Fig. 12. The spectral region of $4340-4800 \AA$ of the high dispersion spectrum.

\subsection{Spectral region $5300-5800 \AA$}

Figure 14 shows the spectral region 5300-5800 $\AA$. The most intense emission line in this region at $5320 \AA$ is identified as

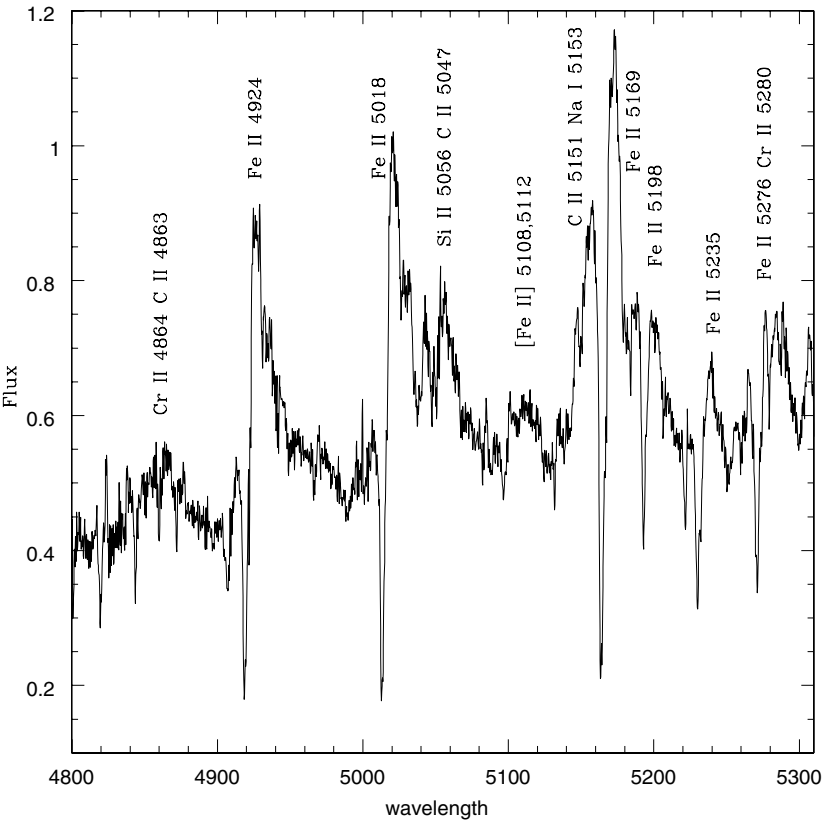

Fig. 13. The spectral region of $4800-5300 \AA$ of the high dispersion spectrum.

a blend of Fe II 48, 5317; Fe II 49, 5317; and Cr II 43, 5313. The narrow absorption lines of Fe II 48, 5363; Fe II 49, 5425; Fe II 56, 5525; Fe II 55, 5535; Fe II 57, 5658; are detected. 


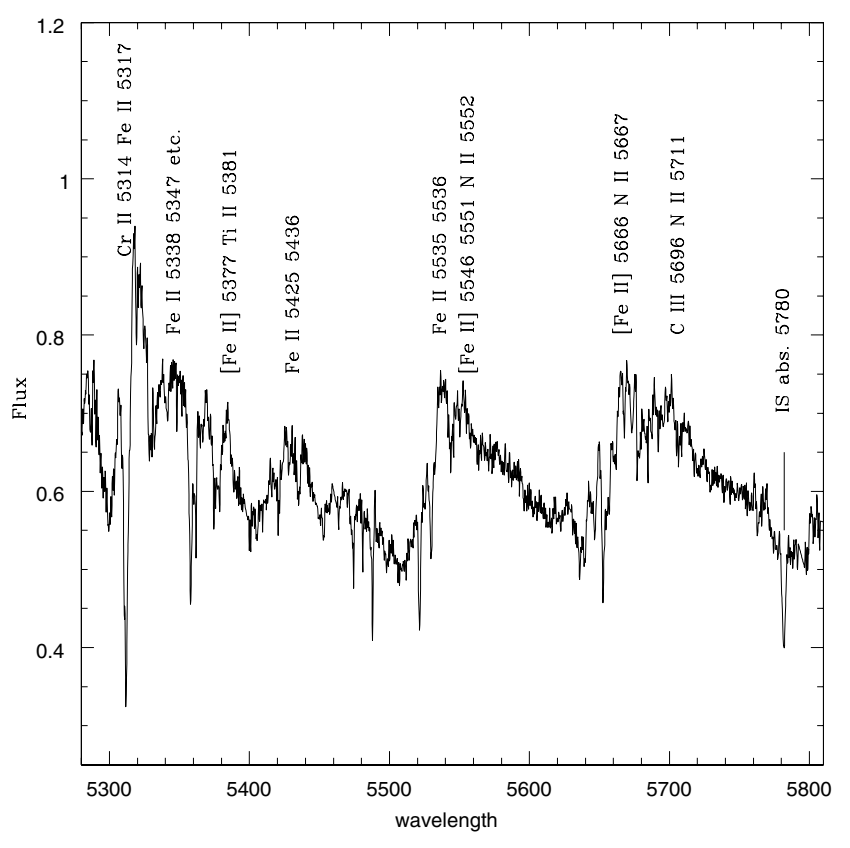

Fig. 14. The spectral region of $5300-5800 \AA$ of the high dispersion spectrum.

Two broad emission complexes are seen at $5550 \pm 25 \AA$ and $5706 \pm 35 \AA$. The emission lines of N II 63, 5535, 5530, 5552, and C II 10, 5536 may have been the main contributors for the former emission complex. The latter emission complex may have consisted of N II 3, 5680, 5667, 5676; C II 15, 5665, 5648, 5641; C III 2, 5696; and some Fe II lines.

An interstellar absorption band is seen at $5780 \AA$. Wagner et al. (2001b) obtained an equivalent width of $1.2 \AA$ for this band. On the other hand, we have measured a much smaller value $0.58 \AA$ in our high dispersion spectra. The reason for the disagreement is unknown.

\subsection{Spectral region $5800-6300 \AA$}

Figure 15 shows the spectral region 5800-6300 A. The most intense emission line in this region is detected at $5900 \AA$. It is without a doubt that the principle contributors to this emission line are both $\mathrm{Na}$ I D1 and D2, which is reported in earlier works (Wagner 2001; Kamath \& Anupama 2002). There may have been, however, some contributions from C II 5, 5890, 5892 and C III 20, 5894 to this emission feature, because emission lines of carbon ions are unusually strong in the spectra of this object. The blue-shifted absorption components of Na I D1 and D2 are detected (see, Sect. 4.6). We will discuss the nature of the interstellar absorption components of Na I D1 and D2 in Sect. 5.

The second most intense emission line is detected at $5877 \AA$. The observed wavelength agrees with that of He I 5876. However, in contrast to the spectra of usual classical novae, He I 5876 may not have been the main contributor to the emission, because the other emission lines of He I at 4471 and $4713 \AA$ are very weak in the same spectrum. The contributions from C III 20, 5858, 5872; and Ca I 46, 5868, in particular, are probably large.

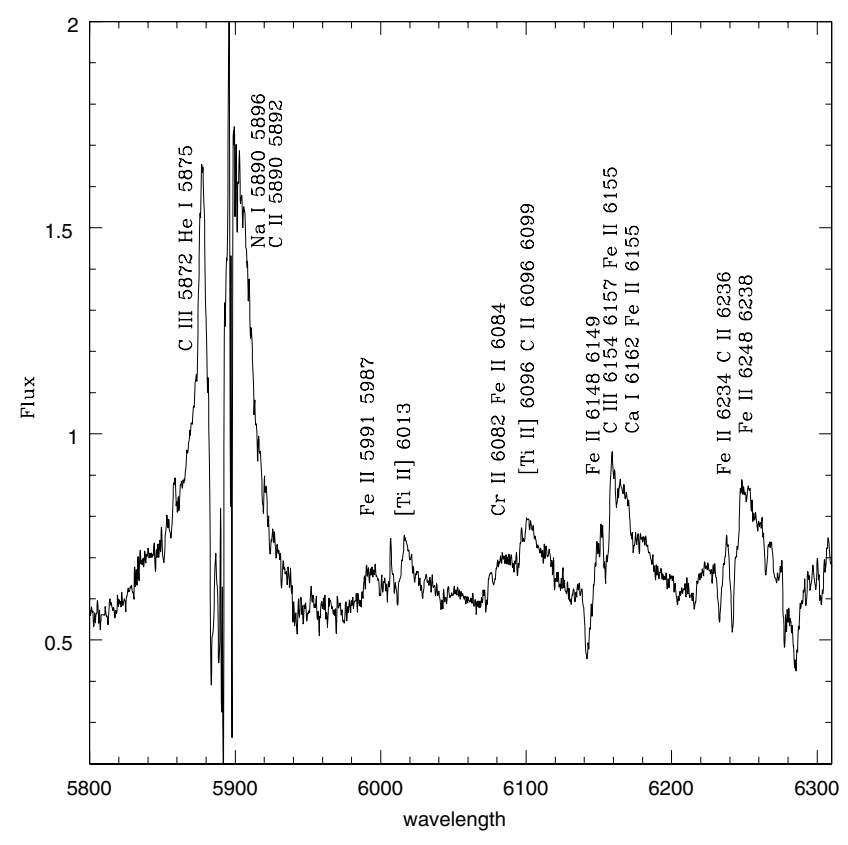

Fig. 15. The spectral region of 5800-6300 $\AA$ of the high dispersion spectrum.

An emission line is detected at $6018.6 \AA$, which has not been observed before in the spectra of typical classical novae (e.g., Williams et al. 1991; Iijima 2006). Even during the robust analysis of Thackeray (1977), of spectra for the symbiotic nova RR Tel, no emission at nearby wavelength was detected. We have identified the detected emission line as [Ti II] 9F, 6013.3. Since this line is not presented in the catalog of Meinel et al. (1968), its appearance in the spectra of astrophysical objects is apparently rare. Weak traces of [Ti II] lines of the same multiplet number at 6047.5 and $6053.1 \AA$ (Moore 1959) were detected (Tables 2, 3), while the lines at 6096.0 and $6087.8 \AA$ were not confirmed, because they were blended with C II 24, 6095.4 and Fe II 46, 6084.1, respectively. The other prominent emission lines in this region are members of Fe II multiplet 74.

\subsection{Spectral region $6300-6900 \AA$}

Figure 16 shows the spectral region 6300-6900 ̊. The expected position of $\mathrm{H} \alpha$ is indicated in the figure, at which, however, no trace of emission nor absorption is seen. This result is consistent with the previous works (Fujii et al. 2001; Wagner et al. 2001a; Kamath \& Anupama 2002). We estimate an upper limit for the equivalent width of $\mathrm{H} \alpha$ emission at $-0.2 \AA$. The strongest emission line shows a double peak profile. The wavelengths of the peaks are $6584 \AA$ and $6588 \AA$, which have been identified as C II 2, 6578 and 6583, respectively. A discontinuity is observed on the blue side of the emission line, which may be due to Ca I 1 , 6573 emission. These identifications are consistent with those of Wagner (2001). On the other hand, most of the unclassified strong Ca I lines given in the multiplet table of Moore (1959) are detected at 6405.9, 6395.2, 5764.3, 5682.9, and 5688.5 (Table 3) which was not reported in the previous works.

There is a broad emission band at $6685 \pm 15 \AA$. The wavelength of its peak is $6682 \AA$ which corresponds to that of He I 6678. This emission band, however, was too intense with 


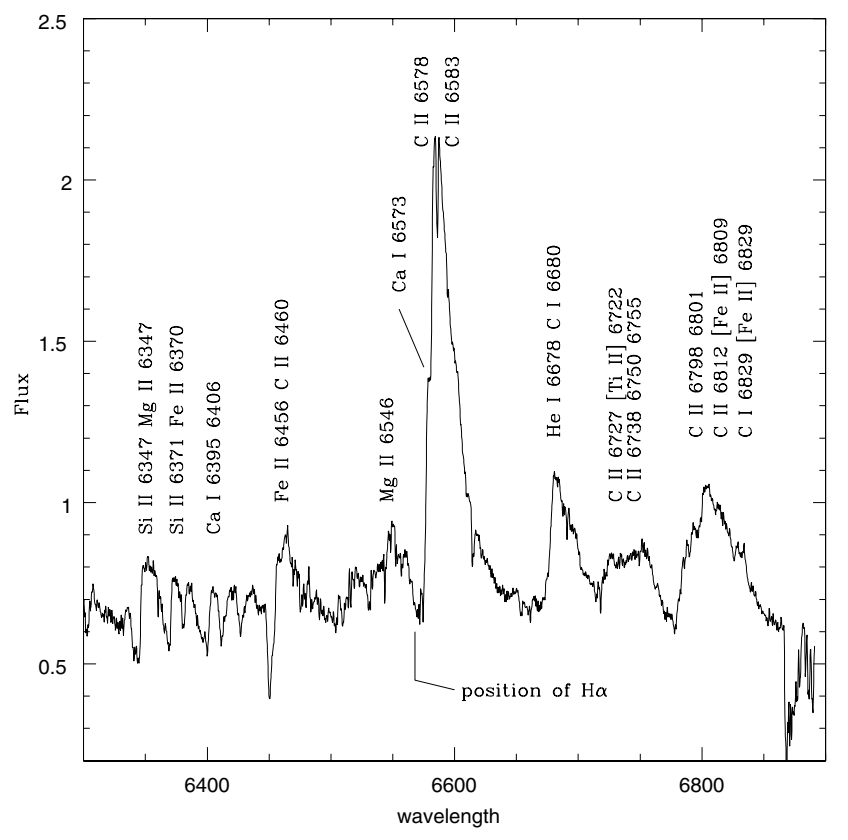

Fig. 16. The spectral region of $6300-6900 \AA$ of the high dispersion spectrum.

Table 4. Radial velocities of selected absorption lines in the early decline stage.

\begin{tabular}{lrrrrrr}
\hline \hline Elements & \multicolumn{5}{c}{ Radial velocity in km s } \\
& Jan. 14 & Feb. 2 & Feb. 3 & Feb. 5 & Feb. 11 & Apr. 2 \\
\hline Ca II H & -310 & & & & & \\
& \pm 40 & & & & & \\
Na I D2 & & & -329.7 & -326.8 & & \\
& & & \pm 4 & \pm 4 & & \\
Na I D1 & & & -358.5 & -358.9 & & \\
& & & \pm 4 & \pm 4 & & \\
metal & & -310.1 & -283.5 & -282.9 & -302.3 & -304.3 \\
& & \pm 10 & \pm 5 & \pm 4 & \pm 5 & \pm 8 \\
\hline
\end{tabular}

Metal: Means of the radial velocities of the absorption components of Fe II, Ti II, and Cr II lines. The FWHMs of the metallic absorption lines are about $210 \pm 5 \mathrm{~km} \mathrm{~s}^{-1}$.

respect to the emission lines of He I 4471 and 4713. There were probably large contributions from C I lines at 6672, 6674, 6680, 6684 , and $6689 \AA$, the wavelengths of which are given in the NIST database. The broad emission complex at $6740 \pm 15 \AA$ may have consisted of the emission lines of C II of multiplet number 21 and [Ti II] 8F, 6725.7 and 6722.0. The emission lines of C II of multiplet number 14 may have been the main contributors to the emission complex at $6810 \pm 20 \AA$.

\subsection{Outflow velocity}

The radial velocity of the system of this object is estimated from the radial velocities of the peaks of prominent emission lines. We selected [Fe II] 19F, 5261.6; [Ti II] 9F, 6013.3; Mg II 23, 6545.8; C II 2, 6578.0; and 6582.9 for this purpose, because these lines were relatively free from blending and were not accompanied by absorption components. The derived radial velocity is $+224 \pm$ $8 \mathrm{~km} \mathrm{~s}^{-1}$, which suggests that this object belongs to the old disk population.

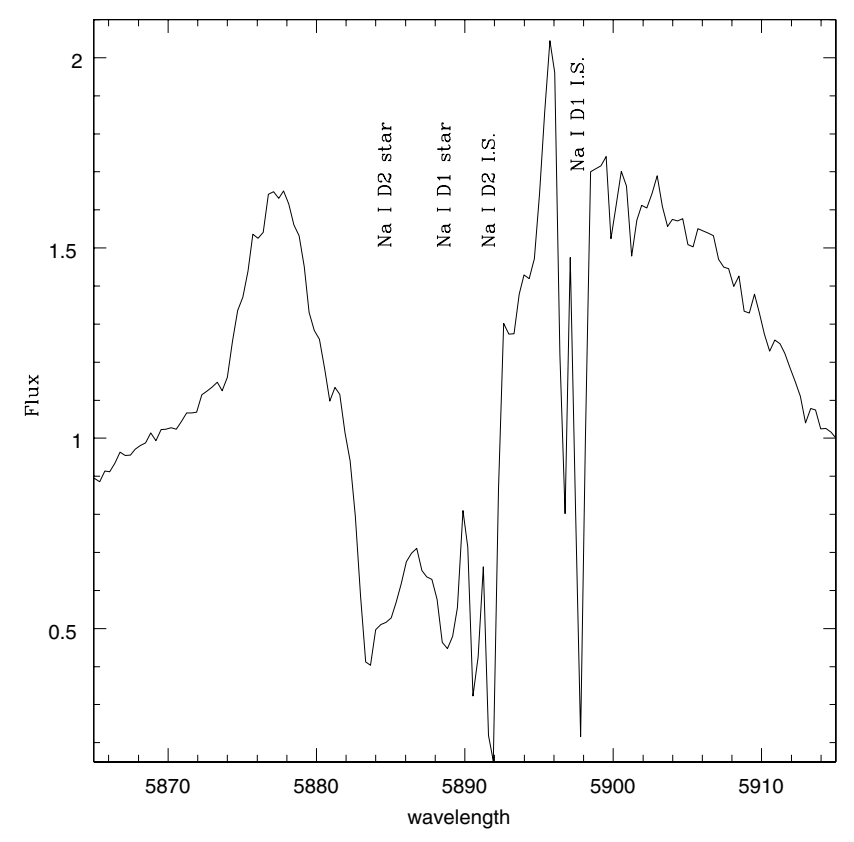

Fig. 17. Profiles of Na I D1 and D2 lines and their interstellar absorption components on February 3, 2001.

The heliocentric radial velocities of the absorption components of selected lines are given in Table 4, where the results obtained with the medium dispersion spectra are also presented. Since the radial velocity of the system is $+224 \mathrm{~km} \mathrm{~s}^{-1}$, the blueshifts of the absorption components were roughly $-500 \mathrm{~km} \mathrm{~s}^{-1}$, and no significant change was noticed during the period of our observations.

The radial velocities of the absorption components of $\mathrm{H} \mathrm{I}$ and $\mathrm{He}$ I were different from those of $\mathrm{Fe}$ II lines in some novae (e.g., Iijima \& Esenoglu 2003; Iijima 2006). It seems that those of $\mathrm{H} \mathrm{I}$ and $\mathrm{He}$ I lines better represent the outflow velocities of the ejecta. Unfortunately, we are unable to find the absorption components of H I or He I in our spectra. The radial velocities in Table 4 may relate to an outflow velocity of the ejecta in a certain stage of the evolution.

\section{Interstellar extinction and distance}

Figure 17 shows a detailed profile of Na I D1 and D2 in the high dispersion spectrum obtained on February 3, 2001. It can be clearly seen that there are two sets of narrow absorption components of Na I D1 and D2. The heliocentric radial velocities of the two components are $+35.3 \pm 0.4$ and $+93.2 \pm 1.0 \mathrm{~km} \mathrm{~s}^{-1}$, which become $+16.0 \pm 0.4$ and $+73.5 \pm 1.0 \mathrm{~km} \mathrm{~s}^{-1}$ in the frame of the local standard of rest. Radio observations for the interstellar emission line of $\mathrm{H} \mathrm{I}$ at $21 \mathrm{~cm}$ in a nearby field $(l=240, b=-2)$ show that there are four prominent emission components with radial velocities of $22,65,77$, and $86 \mathrm{~km} \mathrm{~s}^{-1}$ (Hartmann \& Burton 1997). The $F W H M$ of the emission components are about $10 \mathrm{~km} \mathrm{~s}^{-1}$, that is, the latter three components are seen as one blended emission complex. Since the radial velocities of the narrow absorption components of $\mathrm{Na} I$ lines are close to those due to the interstellar emission of $\mathrm{H} \mathrm{I}$, we have decided that the narrow absorption components are of interstellar origin. Our object, V445 Pup, may be located in or beyond the next outer arm of 
our galaxy, namely the Orion arm in this direction, because two sets of interstellar absorption components are detected.

The equivalent widths of the interstellar absorption components of Na I D 1 are $0.35 \pm 0.02 \AA$ for the lower velocity component and $0.59 \pm 0.01 \AA$ for the higher velocity one. These values are means of those measured in the two high dispersion spectra. Thus, they are slightly different from the values given in Table 3. The equivalent widths of the interstellar absorption components of Na I D2 were not measured, because they were blended with the blue-shifted deep absorption components of $\mathrm{Na}$ I D1 of the stellar spectrum.

One of us (H.N.) estimated the column density of $\mathrm{H}$ I atom in the direction of this object $(l=241.1, b=-2.2)$ using the data of Hartmann \& Burton (1997). The H I radio data were averaged using a weighting function with $F W H M=17 \mathrm{~km} \mathrm{~s}^{-1}$ in order to match the resolution of our spectra. The results are $\log N(\mathrm{H}) \cdot L=$ 20.7 number $\mathrm{cm}^{-2}$ for $v_{\mathrm{lsr}}<16.0 \mathrm{~km} \mathrm{~s}^{-1}$, and $\log N(\mathrm{H}) \cdot L=21.5$ for $v_{\mathrm{lsr}}<73.5 \mathrm{~km} \mathrm{~s}^{-1}$ (see, Nakanishi 2005). Thus, we have $E(B-V)=0.51 \mathrm{mag}$ and $A_{\mathrm{V}}=1.6 \mathrm{mag}$, where the standard relation $N(\mathrm{H}) / E(B-V)=6.2 \times 10^{21} \mathrm{~cm}^{-1} \mathrm{mag}^{-1}$ (Jenkins \& Savage 1974) is used. Our result is consistent with the estimate of Wagner et al. (2001b): $E(B-V) \leq 0.8 \mathrm{mag}$, but is larger than that of Ashok \& Banerjee (2003): $E(B-V)=0.25$ mag.

The interstellar extinction in the field $88(l=240, b=0)$ of Neckel \& Klare (1980) is $A_{\mathrm{V}}<1 \mathrm{mag}$ for $d<3.5 \mathrm{kpc}$, while suddenly increases to $A_{\mathrm{V}}>2 \mathrm{mag}$ at $3.5 \mathrm{kpc}$. On the other hand, the extinction in the field $89(l=242, b=0)$ is $A_{\mathrm{V}}<1 \mathrm{mag}$ for $d<5 \mathrm{kpc}$. Our object is located close to the border between the fields 88 and 89 . Our result, $A_{\mathrm{V}}=1.6 \mathrm{mag}$, suggests that the distance to the object is larger than $3.5 \mathrm{kpc}$, or larger than $5 \mathrm{kpc}$.

If the radial velocity $+73.5 \mathrm{~km} \mathrm{~s}^{-1}$ of the interstellar absorption lines is due to Galactic rotation, the distance to the object should be roughly $6.5 \mathrm{kpc}$, according to the rotation curve of Dehnen \& Binney (1998). Here, we adopt a distance of $3.5 \leq d \leq 6.5 \mathrm{kpc}$. The absolute magnitude at light maximum is $-5.8 \leq M_{\mathrm{V}} \leq-7.1 \mathrm{mag}$ with this distance, where the observed maximum luminosity is $m_{\mathrm{V}}=8.5 \mathrm{mag}$ (Sect. 1).

The theoretical model calculations of Kato \& Hachisu (2003) and Kato (2005) showed that the absolute magnitudes at light maximum of helium novae are about -6 mag in the $V$ band, which is consistent with our result. Recently, Kato et al. (2008) suggested a higher maximum luminosity of a helium nova in their modified model, which agrees with our higher luminosity.

\section{Conclusion}

V445 Puppis has been studied in the past by a number of astronomers. However, its distance or interstellar extinction were not well known. The detection of two sets of the interstellar absorption components of Na I D1 and D2, and their radial velocities could be important clues for the understanding of these properties. Our estimates are $3.5 \leq d \leq 6.5 \mathrm{kpc}, E(B-V)=0.51 \mathrm{mag}$, and $-5.8 \leq M_{\mathrm{V}} \leq-7.1 \mathrm{mag}$, respectively.

The emission lines of Fe II of multiplet numbers, such as $37,38,42,49$, and 74 accompanied by blue-shifted absorption components are typical features in the spectra of Fe II type classical novae (Williams 1992). The emission lines of Ti II, Si II, N II, [Fe II], Cr II, Ca I, Na I, and He I are also familiar in the spectra of classical novae. These spectral features support the classification of this object as a type of novae. On the other hand, the absence of hydrogen lines and the unusually strong emission lines of carbon ions may be characteristic features of this type of object. Some emission lines, which were not observed in the spectra of usual classical novae, were detected in our spectra, for examples, Mg II 23, 6545.8 and [Ti II] 9F, 6013.3. The appearance of $\mathrm{CN}$ molecular absorption band is in addition rare in the spectra of classical novae, and had only previously been observed in spectra of DQ Her. These features may be characteristic of a helium nova. Our results support the classification of this object as a helium nova, but further confirmation is required.

Acknowledgements. We are grateful to Profs. I. Hachisu and M. Kato for the useful discussions and comments, and to Prof. R. Barbon for the careful reading of the manuscript and useful suggestions.

\section{References}

Ashok, N. M., \& Banerjee, D. P. K. 2003, A\&A, 409, 1007

Dehnen, W., \& Binney, J. 1998, MNRAS, 294, 429

Fujii, M. 2001, IAU Circ., 7556

Hartmann, D., \& Burton, W.-B. 1997, Atlas of Galactic Neutral Hydrogen (Cambridge University Press), 243

Iijima, T. 2005, in The Astrophysics of Cataclysmic Variables and Related Objects, ed. J.-M. Hameury, \& J.-P Lasota, ASP Conf. Ser., 330, 441

Iijima, T. 2006, A\&A, 451, 563

Iijima, T., \& Esenoglu, H. H. 2003, A\&A, 404, 997

Jenkins, E. B., \& Savage, B. D. 1974, ApJ, 187, 243

Kato, M. 2005, in The Astrophysics of Cataclysmic Variables and Related Objects, ed. J.-M. Hameury, \& J.-P. Lasota, ASP Conf. Ser., 330, 311

Kato, M., \& Hachisu, I. 2003, ApJ, 589, L107

Kato, M., Hachisu, I., \& Kiyota, S. 2008, in the Proceedings of the conference Hydrogen-Deficient Stars, ed. K. Werner, \& T. Rauch, ASP Conf. Ser., in press

Kato, T. 2000, IAU Circ., 7552

Kamath, U. S., \& Anupama, G. C. 2002, Bull. Astron. Soc. India, 30, 679

Lynch, D. K., Russell, R. W., \& Sitko, M. L. 2001, AJ, 122, 3313

Lynch, D. K., Rudy, R. J., Mazuk, S., Venturini, C. C., Puetter, R. C., \& Perry, R. B. 2004, AJ, 128, 2962

McLaughlin, D. B. 1937, Publ. Obs. Univ. of Michigan, 6, 107

Meinel, A. B., Aveni, A. F., \& Stockton, M. W. 1968, Catalog of Emission Lines in Astrophysical Objects, Optical Sciences Center and Steward Observatory, The University of Arizona, Tucson

Moore, C. E. 1959, A Multiplet Table of Astrophysical Interest, US Dept. of Commerce, Office of Technical Services, Washington DC

Nakanishi, H. 2005, Observational Study in Atomic and Molecular Gas Disks on the Milky Way Galaxy and Nearby Cluster Galaxies, Ph.D. Thesis of the University of Tokyo, Japan

Neckel, Th., \& Klare, G. 1980, A\&AS, 42, 251

Sneden, C., \& Lambert, D. L. 1975, MNRAS, 170, 533

Thackeray, A. D. 1977, Mem. R. astr. Soc., 83, 1

Wagner, R. M. 2001, http://vela.as.arizona.edu/ rmw/v445pup.html Wagner, R. M., Foltz, C. B., \& Starrfield, S. G. 2001a, IAU Circ., 7556

Wagner, R. M., Schwarz, G., \& Starrfield, S. G. 2001b, IAU Circ., 7571

Williams, R. E. 1992, AJ, 104, 725

Williams, R. E., Hamuy, M., Phillips, M. M., et al. 1991, ApJ, 376, 721

Woudt, P. A., \& Steeghs, D. 2005, in The Astrophysics of Cataclysmic Variables and Related Objects, ed., J.-M. Hameury, \& J.-P Lasota, ASP Conf. Ser., 330, 451 